\title{
Codeswitching auf einem hochdiversen urbanen Wochenmarkt: Kommerz, Kommunikation und Identität
}

\author{
Serkan Yüksel (Berlin) und İrem Duman (Berlin)
}

\begin{abstract}
Today's European cities exhibit a great cultural and linguistic diversity. Highly diverse urban areas bring together people from different sociolinguistic backgrounds and thus facilitate intense language contact, with speakers accessing diverse linguistic resources that they creatively use and mix (cf. e. g. Wiese 2020; Otsuji/Pennycook 2010; Pennycook/Otsuji 2015). Such linguistic practices include code-switching.

In previous research on code-switching the focus was on relatively homogeneous settings, mostly bilingual communities (cf. Poplack 2015; Torres Cacoullos/Travis 2015; Bullock/ Toribio 2009). As a part of a larger project, we collected spontaneous speech data through audio and video recordings from a highly diverse street market in Berlin-Neukölln that is popular among locals and tourists, the "Maybachufer-Markt".

The analysis of our data reveals new insights with respect to sociolinguistic motivations underlying code-switching. In light of commercial intentions, vendors try to switch to a language according to how they construct the customers' identity (cf. Bucholtz/Hall 2005; Pfaff-Czarnecka 2011). Besides vendors also commodify specific languages or multilingualism (Heller 2010) based on their and others' language attitudes or they switch to a language for the purpose of maintaining the communication in sales conversations. Correlating these different factors, we will argue that code-switching is used with a commercial motivation in interactions between vendors and customers.
\end{abstract}

\section{$1 \quad$ Einleitung}

Urbane Gebiete in Europa sind heute durch ihren heterogenen und hochdiversen Charakter eine Quelle sprachlicher Innovation. Auch in Großstädten im deutschsprachigen Raum lässt sich eine große ethnografische und sprachliche Diversität beobachten. Ein Beispiel für einen hochdiversen urbanen Raum ist der Maybachufer-Wochenmarkt in Berlin-Neukölln, der Menschen mit ganz unterschiedlichen linguistischen Hintergründen zusammenbringt und dadurch einen intensiven und vielschichtigen Sprachkontakt begünstigt. In solchen urbanen Kontexten stehen Sprecher:innen verschiedene sprachliche Ressourcen und Optionen zur Verfügung, die sie kreativ nutzen und mischen können (cf. auch Wiese 2020; Otsuji/Pennycook 2010; Pennycook/Otsuji 2015; Duman/Lin 2021). 
Basierend auf Daten, die im Rahmen eines größeren Forschungsprojekts ${ }^{1}$ erhoben wurden, haben wir für eine umfassende Analyse des Marktes zwei Blickwinkel eingenommen, die sich gegenseitig bedingen und ergänzen: Einerseits wurde durch die Erfassung ethnografischer Gegebenheiten das soziale Setting des urbanen Raums festgestellt und andererseits wurden durch die Analyse des Sprachgebrauchs die linguistischen Eigenschaften dieser räumlichen Konfiguration untersucht.

Von dieser biperspektivischen Herangehensweise profitierte insbesondere die Untersuchung zu Codeswitching (kurz: CS), das sich auf dem Markt auf interessante Weise vom CS in bilingualen Sprecher:innengruppen unterscheidet. Ausgehend von den räumlichen Merkmalen und der durch diesen Raum begünstigten interaktionalen Rollen der Verkäufer:innen und Kund:innen wurde beobachtet, dass dem CS eine soziolinguistische Motivation zukommt, die sich unter anderem in der Schnittmenge zwischen Kommerz, Kommunikation und Identitätskonstruktion bewegt: Basierend auf ihren Annahmen über die soziale und insbesondere ethnolinguistische Zugehörigkeit der Kund:innen versuchen Verkäufer:innen Elemente einer Sprache zu benutzen, die sie mit dieser Zugehörigkeit in Verbindung bringen. Das Sprachwissen der Verkäufer:innen kann sich auf diese für den Verkaufsakt relevanten Elemente begrenzen, weshalb sich das Ereignis auf der sprachlichen Oberfläche oft in Form von CS zwischen diesen und einer anderen ergänzenden Sprache manifestiert. Diese besondere Art des CS erweist sich in der Verkaufsinteraktion als effektives Mittel, Kund:innen anzuwerben oder vom Kauf zu überzeugen. Beim Switchen in eine bestimmte Sprache oder bei der Produktion mehrsprachiger Ausdrücke können auch die eigenen Spracheinstellungen der Verkäufer:innen und angenommene Spracheinstellungen über die Kund:innen eine Rolle spielen. Manchmal hängt das CS aber auch nur von dem essenziellen Faktor der Gewährleistung der Kommunikation ab.

Wir diskutieren in unserem Beitrag Befunde zu diesen Arten von CS, betten sie in den Hintergrund urbaner Sprachenvielfalt und der Interaktion auf hochdiversen Märkten ein und argumentieren dafür, dass sie kommerziell motiviert sind.

In den Präliminarien wird kurz darauf eingegangen, womit sich die bisherige Forschung zu CS beschäftigt hat und wie sich CS auf dem Maybachufer-Markt davon unterscheidet. Auf diese erste Analyse wird die Hypothese aufgebaut, dass dem CS auf dem Markt eine kommerzielle Motivation zugrunde liegen kann (Kapitel 2). Nach der Besprechung der Daten und Methoden in Kapitel 3, beleuchten wir in den darauffolgenden Kapiteln das Phänomen des kommerziellen CS aus drei verschiedenen Perspektiven. In Kapitel 4 gehen wir auf die Rolle der Identitätskonstruktion ein, der sich Verkäufer:innen bedienen, um in die Sprache, die als die Sprache der Kund:innen interpretiert wird, zu switchen. Der daraus resultierende und für den Verkaufsakt positive Effekt wird vor dem Hintergrund von Face-Theorien diskutiert. In Kapitel 5 werden Beispiele angeführt, in denen eine spezifische Sprache oder die Mehrsprachigkeit zum Instrument der Werbung gemacht wird. Dabei spielen Spracheinstellungen eine wichtige Rolle. Das Phänomen wird im Rahmen der Sprachkommodifizierung bewertet. In Kapitel 6 werden Beispiele angeführt, die weder einer Identitätskonstruktion seitens der Verkäufer:innen zugeordnet werden können, noch direkt mit der Sprachkommodifizierung im Zusammenhang zu sein

\footnotetext{
1 Teilprojekt A01 des SFB 1287 „Limits of Variability in Language“, Universität Potsdam (gefördert durch die Deutsche Forschungsgemeinschaft (DFG)); Projektnummer 317633480.
} 
scheinen. Es geht bei diesen Beispielen schlicht darum, mit dem Gegenüber eine Kommunikation aufzubauen bzw. verstanden werden zu können. In Kapitel 7 wird die Konstitution des kommerziellen CS aus der Perspektive der drei Faktoren, die in den Kapiteln 4-6 beschrieben worden sind, und die Grenzen zu anderen Arten von CS auf dem Markt diskutiert.

\section{Präliminarien: Codeswitching auf dem Maybachufer-Markt}

Diese Arbeit beschäftigt sich mit soziolinguistischen Motivationen für Sprachwechselphänomene, die in Interaktionen zwischen Verkäufer:innen und Kund:innen auf dem Maybachufer-Markt auftreten. Genauer wird versucht für eine kommerzielle Motivation $\mathrm{zu}$ argumentieren, die den Verkaufsakt zu begünstigen scheint. Mit der Analyse wird ein Beitrag zur Forschung von Sprachwechselphänomenen angestrebt.

Sprachwechselphänomene finden in der linguistischen Forschungsliteratur intensive Berücksichtigung aus unterschiedlichen Perspektiven und dementsprechend heterogen sind auch die eingeführten Termini. „Codeswitching“ oder „Codemixing“ sind die häufigsten Begriffe, die solche Phänomene beschreiben (Boztepe 2002; Lim/Ansaldo 2016). „Codeswitching“ referiert auf die Alternation von Varietäten innerhalb einer Konversation, gewöhnlicherweise an zwei semantisch oder soziolinguistisch bedeutungsvollen Junkturen, welches wiederum mit bestimmten pragmatischen Effekten, Diskursfunktionen oder Gruppenidentitäten assoziiert wird (Lim/Ansaldo 2016). Einige Forscher:innen benutzen „Codeswitching“ für den Wechsel von Sprachen oder sprachlichen Varietäten zwischen Phrasen im Kontrast zu „Codemixing“, welches auf den Wechsel innerhalb einer Phrase referiert (Kachru 1978, 1983; Singh 1985; Sridhar/Sridhar 1980). Um dieser Unterscheidung zu entgehen führt Auer (1995) den Begriff der „Codealternation“ ein. Andere benutzen „Codeswitching“ als Überbegriff und spezifizieren strukturelle Unterschiede wenn nötig mit Attributen wie ,inter-,, oder „intraphrasal“ (Milroy/Muysken 1995; Myers-Scotton 1993; Boztepe 2002; Gardner-Chloros 1991; Gumperz 1996).

Der letztgenannten Literatur folgend, verwenden wir „Codeswitching“ (CS) und dazugehörend das Verb switchen für alle Arten von Sprachwechselphänomenen in unseren Daten, unbeachtet ihrer strukturellen Eigenschaften. Gumperzs (1996: 365) Definition von CS als ,alternation among different speech varieties within the same event" und Myers-Scottons (1993: 1) Definition als ,alternations of linguistic varieties within the same conversation“ folgend, beziehen wir uns dabei nicht nur auf einzelne Phrasen und Sätze, die von einer Person produziert wurden, sondern auf ganze zusammenhängende Gespräche.

Der Fokus der CS-Forschung liegt stark auf situationellen und kontextuellen Motivationen des Switchens. Eine der Pionierarbeiten in dem Feld von Blom/Gumperz (1972) untersucht eine Gemeinde im Norden Norwegens, Hemnesberget, und zeigt wie Ranamål, ein lokaler Dialekt, eine lokale kulturelle Identität symbolisiert und mit Heimat, Familie und Freundschaften sowie lokal basierten Aktivitäten und Beziehungen assoziiert wird. Bokmål, also die Standardvarietät des Norwegischen, wird mit Bildung und offiziellen sowie geschäftlichen Angelegenheiten, Religion und den landesweiten Medien assoziiert. Die Sprecher:innen switchen innerhalb eines Gespräches je nach thematischem Zusammenhang von einer Varietät in die andere. So kommt es vor, dass ein Bankbesucher den Bankangestellten, den er aus seinem sozialen Umfeld kennt, auf Ranamål begrüßt und einen Smalltalk mit ihm führt, aber ins Bokmål switcht, sobald es um 
das Bankgeschäft geht. In einer späteren Arbeit betont Gumperz (1982) die Rolle des CS, um einen konversationellen Kontext zu definieren. Myers-Scotton (1993) schreibt, dass es in jeder multilingualen Community eine markierte und eine unmarkierte Art des Sprachwechsels gibt, also dass die benutzte Sprache erwartet oder unerwartet vor dem Hintergrund eines bestimmten Kontextes ist. So ist in Nairobi, der Hauptstadt Kenias, Swahili eine offizielle Amtssprache, aber auch eine Verkehrssprache für alle, die nicht dieselbe Erstsprache (L1) sprechen. MyersScotton (1993: 88) gibt ein Beispiel, in dem ein Sprecher beim ersten Kennenlernen in einer Arbeitsstätte mit dem Angestellten zunächst auf Swahili spricht, bis sich im Gespräch herausstellt, dass die Interaktanten derselben ethnischen Gruppe zugehören. Daraufhin switchen sie ins Luyia, ihre ethnische Sprache, und markieren damit ihre gemeinsame Identität. CS kann als markiert wahrgenommen werden, wenn es den erwarteten Mustern widerspricht (cf. Myers-Scotton 1993). Myers-Scotton (2006: 162) führt dazu ein Beispiel an, bei dem ein Fahrgast den Busfahrer auf Luyia anspricht, um die geteilte Gruppenzugehörigkeit zu markieren. Damit möchte er einer gewöhnlichen Bezahlung des Fahrschein ausweichen. Die unmarkierte Sprachwahl in der Situation wäre Swahili.

In der Literatur werden neben diesen thematischen oder solidaritätsmarkierenden Motivationen und Funktionen von CS noch weitere soziolinguistische Funktionen beschrieben, die mit Identitätsmarkierung, Affektivität oder Metaphorik assoziiert werden (Holmes 2013; GardnerChloros 2009; Gumperz 1982; Winford 2003). Daneben gibt es jedoch auch Ansätze, die sich von dieser diskursorientierten Perspektive abgrenzen. Kaschubat (2004, zitiert nach Peterson 2016: 118) motiviert das CS in ihren russisch-deutschen Daten mit informationsstrukturellen Gründen. Demnach sind 90\% der Ein-Wort-Switches in ihren Daten fokusmarkiert. Die „communication accomodation theory“ von Giles (1978) nimmt eine sozialpsychologische Perspektive ein und konzentriert sich auf die Rolle von Konvergenz- und Divergenzprozessen beim Aufbau von Beziehungen zwischen Gruppen. CS wird aus der Perspektive von Handlungen zum Identitäts- und Publikumdesign analysiert. Der Fokus liegt nicht auf einzelnen Turns bzw. Switches, sondern auf dem gesamten Gespräch (cf. Muysken 2011).

Eine andere Herangehensweise sehen wir in der Literatur, wenn es um das „mixed code as default" geht, so wie es Lim und Ansaldo (2016) theorienunabhängig benennen. Eine solche Situation liegt dann vor, wenn sich Sprachalternation als eine Default-Option in einer Sprecher:innengruppe gefestigt hat und eine geteilte mehrsprachige Identität konstruiert. Meeuwis/Blommaert (1998) zeigen das beispielsweise für Lingala-Französisch-Sprecher:innen in Belgien. Auer (1999) beschreibt ein Kontinuum von Sprachmisch- oder -wechselphänomenen und kategorisiert diese Art als „fused lects“. Diese Art des für bilinguale Sprecher:innengruppen typische CS wird in den bisherigen Forschungen ausgiebig beschrieben und analysiert. ${ }^{2}$ Was diese Arbeiten gemeinsam haben ist, dass sie Daten beschreiben, in denen sich eine distinkte Motivierung einzelner Switches als schwer oder gar unnötig zeigt. Sie sprechen oft von „bilingualen Sprachcommunitys“, deren Mitglieder zwei Sprachen meist auf vergleichbaren Niveaus beherrschen. Eine der Sprachen gilt dabei oft als Hauptverkehrssprache des Landes und die andere als Heritage-Sprache oder die Sprache einer ethnischen Gruppe.

\footnotetext{
${ }^{2}$ Nur um ein paar Beispiele für die Forschung von CS in bilingualen Sprecher:innengruppen zu nennen: Für Deutsch-Türkisch Kallmeyer/Keim 2003; Özdil 2010; Çetinoğlu 2017; für Niederländisch-Türkisch Backus 1996; für Englisch-Spanisch Poplack 1980; für Englisch-Inuktitut Allen 2009.
} 
Der Begriff der „Sprachcommunity“ bzw. der „,bilingualen Sprachcommunity“ zeigt sich in der Literatur teils als kontrovers und teils als definitionslos, da die Fragen, wo die Grenzen einer solchen Community gesetzt werden und inwieweit man von einer Gemeinschaftlichkeit sprechen kann, oft unbeantwortet bleiben (cf. Patrick 2004; Rampton 2009). Vor diesem Hintergrund wird in der vorliegenden Studie der Begriff „,bilinguale Sprecher:innengruppen“ bevorzugt, der zunächst alle Sprecher:innen jenseits ihrer Vernetzung und sprachlichen Historie, ungeachtet von Erwerbsalter und -form der einzelnen Sprachen, zusammenführt, die zwei Sprachen mehr oder weniger fließend sprechen können. Besonders wichtig für unsere Daten ist die Kombination Deutsch-Türkisch und Deutsch-Arabisch, da die Verkäufer:innen aus den Beispielen jeweils zu einer dieser Sprecher:innengruppen gezählt werden können. Die Zugehörigkeit $\mathrm{zu}$ einer bilingualen Sprecher:innengruppe soll nicht ausschließen, dass Verkäufer:innen weitere Sprachen auf einem nicht fließenden Niveau beherrschen.

Statt von CS spricht Canagarajah (2009) von „,plurilingualen Praktiken“ und andere von „Translanguaging“ (García 2009a, 2009b; Blackledge/Creese 2010; Li 2011, 2017). In der gegenwärtigen Literatur lassen sich noch andere Begriffe wie „translingual practice“ (Canagarajah 2013), „transglossia“ (García 2013, 2014; Sultana et al. 2015), ,polylingual languaging“ (Jørgensen 2008a, 2008b; Møller 2008), „metrolingualism“ (Otsuji/Pennycook 2010; 2014), „flexible bilingualism“ (Creese/Blackledge 2011), „multilingua“ oder „metrolingua francas“ (Makoni/Pennycook 2012; Pennycook/Otsuji 2014) und „language crossing“ (Rampton 2017) finden, die sich jeweils nur in kleinen Bedeutungsnuancen unterscheiden oder sich mit unterschiedlichen Kontexten beschäftigen. Ein großer perspektivischer Unterschied ist, dass diese Arbeiten das Geschehen im Gegensatz zu CSAnsätzen primär aus der Perspektive eines durch alle Varietäten gebildeten Repertoires eines Sprechers, einer Sprecherin oder beim metrolingualism eines urbanen Settings betrachten. Sprachen als separate Systeme sind nur sekundär von Bedeutung oder ihre Existenz als solche in der menschlichen Kognition werden in einigen dieser Ansätze sogar gänzlich in Frage gestellt. Einen Überblick über einige dieser Ansätze bietet Pennycook (2016) und eine kritische Auseinandersetzung MacSwan (2017), der vorliegenden Studie sind etablierte Ansätze aber nur teilweise dienlich. Zum einen nehmen die Verkäufer:innen auf dem Markt Sprachen als mehr oder minder separate Systeme wahr, die sie als solche einsetzen. Das zeigt sich in den Fokusgruppeninterviews ${ }^{3}$ mit den deutsch-türkischsprachigen Verkäufer:innen (1). ${ }^{4}$
01 V_KA_03:
Wir sagen Sachen auch in anderen Sprachen.
02 V_BR_01:
03 V_KA_01: Gute Frage Bruder $<$ Name von V_BR_01>, Italienisch, Spanisch, ,bye bye“ zum Beispiel.
04 V_KA_03: Manchmal Japanisch oder so ((...)) Arabisch oder meistens Englisch.

\footnotetext{
${ }^{3}$ Fokusgruppeninterviews sind Interviews mit den Verkäufer:innen, die nach den audiovisuellen Aufnahmen der Verkaufsinteraktionen durchgeführt wurden. So gab es nach der ersten Analyse der Verkaufsgespräche die Gelegenheit, weitere Unklarheiten oder Forschungsfragen zu klären. Details zu den Daten und Methoden folgen in Kapitel 3.

${ }^{4}$ Bei allen in der vorliegenden Arbeit aufgeführten Ausschnitten aus den Fokusgruppeninterviews handelt es sich um Übersetzungen aus dem Türkischen. Erklärungen von uns stehen in Winkelklammern <Erklärung>. Bei doppelten Winkelklammern steht in den äußeren Klammern das Gesagte und in den inneren Klammern unsere Erklärung zum Gesagten. Da das Türkische keine Genus- bzw. Geschlechtsmarkierung kennt und Begriffe sich geschlechtsneutral auf alle Personen beziehen können, wurde bei der Übersetzung gegendert.
} 
05 V_OG_03: Arabisch, Englisch, Deutsch.

Zum anderen geht es in der vorliegenden Studie um eine kommerzielle Motivation hinter dieser bewussten Sprachwahl von Verkäufer:innen, die sich auf der Oberfläche in Form von CS manifestiert. Arbeiten mit Translanguaging-Ansätzen beschäftigen sich bis dato weniger mit motivationalen Faktoren einzelner Switches, sondern konzentrieren sich meistens auf eine ganzheitliche Darstellung eines Settings. Für den Markt besonders interessant ist der metrolingualism-Ansatz, der einen urbanen Raum als Quelle des sprachlichen Inputs definiert (Otsuji/Pennycook 2010; Pennycook/Otsuji 2015). Eine wichtige Frage in diesem Zusammenhang, die wir den Verkäufer:innen gestellt haben, ist, woher ihre sprachlichen Ressourcen stammen, wo sie die Sprachen, die sie in dem in (1) dargestellten Gesprächsausschnitt aufgezählt haben, erworben haben, ob sie beispielsweise einen Sprachkurs besucht haben oder nicht (2).
01 V_OG_01:
02 V_OG_03:
03
Nein $<$ hat keine Sprachkurse besucht $>$, auf dem Markt.
04 V_KA_01:
Durch das Lauschen hier auf dem Markt oder durch die Mitverkäufer:innen. Man lernt eine Sprache am besten draußen und nicht in einer Schule.
05
Wir versuchen unsere Arbeit mit dem laufen zu lassen, was wir durchs Lauschen
06 erfassen. Es soll praktisch und schnell gehen. Es reicht, wenn wir verstehen, was gekauft werden soll. Wenn ich dann noch den Preis nennen kann, okay, das genügt.

Der Markt selbst also gibt den nötigen sprachlichen Input zum Erwerb dieser Sprachen. Um es in den Worten von Pennycook und Otsuji zu fassen, bildet auch der Maybachufer-Markt einen Ort mehrstufiger Mehrsprachigkeit, wo verschiedene sprachliche Ressourcen mobilisiert werden (cf. Pennycook/Otsuji 2015).5 Diese mehrstufige Mehrsprachigkeit (,layered multilingualism“) zeichnet sich dadurch aus, dass der Spracherwerb auf bestimmte Bereiche wie Preise, Zahlen, Begrüßungen und einfache Floskeln oder Produktbezeichnungen begrenzt sein kann, wie teilweise aus (2:05-06) zu erkennen ist.

Es ist nicht einfach, die hohe Diversität der sprachlichen Varietäten abzubilden, die diese sprachlichen Ressourcen konstituieren, da der Markt von Menschen mit sehr unterschiedlichen soziolinguistischen Hintergründen besucht wird. Einen Richtwert geben uns die soziolinguistischen Kurzinterviews mit den Marktbesucher:innen, bei denen 224 Kund:innen 45 verschiedene Sprachen als ihre L1 angegeben haben. In den audiovisuellen Aufnahmen der Verkaufsinteraktionen, aus denen die CS-Beispiele in der vorliegenden Studie stammen, haben wir an verschiedenen Stellen die Benutzung von zwölf dieser Sprachen feststellen können (siehe Kapitel 3 für die hier angegebenen Daten und Methoden und für Details zu den Sprachen). In (3) ist ein zusammenhängendes Verkaufsgespräch mit Arabisch, Deutsch, Englisch, Hebräisch, Italienisch und Türkisch dargestellt, das zeigt, was ein Resultat dieser Diversität sein kann. ${ }^{6}$

\footnotetext{
${ }^{5}$ Pennycook/Otsuji (2015: 177): „Markets are sites of layered multilingualism in which different language resources are mobilized within a flow of other practices.“

${ }^{6}$ Transkriptionskonventionen für die CS-Beispiele: Nach der Beispielnummer folgt die Interaktionsart: VG steht für Verkaufsgespräch, MS für Marktschreien und PG für Privatgespräch. Die Eigenschaften dieser Interaktionsarten werden im weiteren Verlauf von Kapitel 2 erklärt. Bei den Siglen der Interaktant:innen steht K für Kund:innen und V für Verkäufer:innen. Eine genauere Erklärung für die Siglen folgt in Kapitel 3. Die im Gespräch verwendeten Sprachen werden in Form von ISO-639-3-Kürzeln in der ersten Zeile alphabetisch angegeben. Schrifteinstellungen wie Kursivschrift und Unterstreichungsarten sollen verdeutlichen, an welcher Stelle welche Sprache
} 


$$
\text { VG }[\mathrm{ara}][\mathrm{deu}][\underline{\mathrm{eng}}][\underline{\underline{\mathrm{heb}}}][\mathrm{ita}][\underline{\mathrm{tur}}]
$$

01 K_KA_01 how much is it?

02 V_KA_01 ehm(-) sechs fünfzig(-) six euro forty cent.

03 K_KA_01 six euro? (.) six euro?

04 V_KA_01 sechs euro fifty cent(.) sechs fünfzig (-)

05 K_KA_01 nein israel. ((...))

06 V_KA_01 israil ehm $(-) \quad<<$ spricht mit Vornamen V_BR_01 an $><$ Name $>$

$\underline{a b i}$

Bruder

08

09

V_BR_01

0 V_KA_01

11 K KA 01

12 V KA 01

13 K_KA_01

14 V_KA_01

15 K_KA_01

16 V KA 01

17 K KA 01

sechs

\section{arapça-da}

Arabisch-LOC elli ne?>

fünfzig was

,Bruder $<$ Name $>$, was war 6,50 nochmal auf Arabisch, was ist 6,50 auf Arabisch?

sitte euro nus.

sechs Euro halb

$<<$ antwortet K_KA_01> sitte euro nuṣ.>

((lacht))

AUCH nicht?

wir haben nicht arabisch.

nicht arabisch?

hebräisch.

hebRÄisch(.) ah (-) noch schlimmer(.) das könnwa nicht.

hebräisch ist eh (-) shesh vahetsy.

sechs und.halb italiano?

italienisch

ne-ydi?

was-PAST $\overline{\text { Arabisch-LOC }}$

18 V_KA_01 <<wiederholt> shesh vahetsy. $>$

Dieses vergleichsweise kurze Gespräch wirft interessante Fragen bezüglich der Motivation hinter den verschiedenen CS-Phänomenen auf. Der Kunde fragt auf Englisch nach dem Preis eines Produktes (3:01), die Verkäuferin antwortet auf Deutsch und switcht ins Englische (3:02). Das Switchen der Verkäuferin ins Englische interpretieren wir in diesem Beispiel mit einer rein kommunikativen Motivation, der Kunde fragt nämlich auf Englisch und könnte Deutsch

verwendet wird. Die Transkriptionen sind ein- bis dreispurig aufgebaut. In der ersten Spur folgt die Transkription der aufgenommenen Sprache zur besseren Erkennung in fetter Schrift und in der Schriftart Courier, in der zweiten Spur eine Glossierung, die für alle Sprachen außer Deutsch und Englisch vorgenommen wird. An einigen Stellen folgt in der dritten Spur in Anführungszeichen eine idiomatische Übersetzung bzw. Übertragung. Diese Übersetzung soll ein besseres Verständnis von Stellen ermöglichen, die für Leser:innen nicht auf den ersten Blick verständlich sein könnten. Generell orientieren wir uns an den GAT2-Basiskonventionen für Transkriptionen (Selting et al. 2009). Die Nummerierung bezieht sich nur auf die Transkription der gesprochenen Sprache, also auf die erste Spur. Einfache Klammern mit (.), (-), (--) und (---) beziehen sich ihrer Länge entsprechend auf Sprechpausen. Einfache Klammern ohne Inhalt ( ) geben unverständliche Stellen und mit Inhalt (beispiel) einen vermuteten Wortlaut wieder. Mögliche Alternativen werden mit einem Schrägstrich (/alternative) wiedergegeben. Doppelte einfache Klammern zeigen nonverbale Kommunikation auf, z. B. ((hustet)). Sprachbegleitete nonverbale Kommunikation wird mit doppelten Winkelklammern gekennzeichnet. Im inneren Klammernpaar steht dabei die nonverbale Handlung und im äußeren die Sprache, z. B. $<<$ hustend $>$ hallo $>$. Aber auch zusätzliche Erklärungen oder Informationen zum Gesagten können in Winkelklammern angegeben werden, beispielsweise anonymisierte Namen mit $<$ Name $>$. Sich überlappende Stellen von verschiedenen Sprecher:innen werden mit eckigen Klammern [ ] gekennzeichnet, wobei die sich überlappenden sprachlichen Elemente Zeile für Zeile untereinander stehen. Im Gegensatz zu den GAT2-Basiskonventionen haben wir phonologischen Eigenschaften keine Beachtung geschenkt, da sie für die vorliegende Arbeit keine Rolle spielen. So wird nur ein extra starker Akzent mit Großbuchstaben kenntlich gemacht. Bei der Tonhöhenbewegung wird nur ein steigender Ton mit einem Fragezeichen gekennzeichnet, allen weiteren Tonhöhenverläufen folgt ein Punkt. ((..)) deutet auf eine Auslassung im Transkript. 
womöglich nicht verstehen. Im späteren Verlauf des Gesprächs wird sich aber zeigen, dass er Deutsch teilweise verstehen und produzieren kann, weshalb das Deutsche das Englische verdrängt. In der Tat zeichnen sich die beiden Sprachen als Verkehrssprachen des Marktes in einem dichotomen Verhältnis zueinander ab. Die Verkäufer:innen und lokalen Besucher:innen des Marktes benutzen mehrheitlich das Deutsche zur Kommunikation, wenn es keine gemeinsame Heritage-Sprache wie Türkisch oder Arabisch gibt. Besucher:innen, die Englisch besser als Deutsch sprechen oder gar kein Deutsch sprechen, benutzen meist Englisch für die Kommunikation, meist sind das internationale Tourist:innen. Da die Verkäufer:innen, nach ihren eigenen Angaben (siehe Kapitel 3) Englisch nicht so fließend sprechen wie Deutsch, zeigt sich die von ihnen produzierte Sprache oft in Form von CS zwischen den beiden Sprachen.

In (3:04) fragt die Verkäuferin auf Italienisch, ob der Kunde Italiener sei. Ein gewisses äußerliches Erscheinungsbild des Kunden verleitet die Verkäuferin seine Identität zu konstruieren und führt zu der Annahme einer sozialen Zugehörigkeit des Kunden zu Italien. Diese Annahme erweist sich jedoch als falsch und der Kunde gibt an, dass er eine Zugehörigkeit zu Israel hat (3:05). Aus ihrem vorigen Weltwissen kennt sie wahrscheinlich Menschen aus Israel, die Arabisch sprechen und möchte dem Kunden, obwohl er es schon verstanden hatte, den Preis noch einmal auf Arabisch sagen. Sie fragt einen Verkäufer von einem anderen Stand, der wie sie zur deutsch-türkischen Sprecher:innengruppe gehört, wie man den Preis auf Arabisch sagt (3:06-08). Sie weiß anscheinend, dass der andere Verkäufer Preisausdrücke auf Arabisch produzieren kann. Sie leitet den neu erworbenen arabischen Ausdruck an den Kunden weiter (3:10), woraufhin der Kunde lacht und sagt, dass Hebräisch die passende Sprache wäre (3:11-15). Er gibt ihr einen Input auf Hebräisch (3:17), den sie reproduziert (3:18). Der Switch ins Türkische in der Kommunikation zwischen den beiden Verkäufer:innen kann mit ihrer Zugehörigkeit zu einer deutsch-türkischen Sprecher:innengruppe erklärt werden. Viel interessanter ist jedoch, dass die Verkäuferin ins Italienische, Arabische und Hebräische switcht. Sie selber identifiziert sich nicht mit diesen Sprachen, die Switches sind auch nicht unabdingbar für die Kommunikation wie etwa das Switchen ins Englische am Anfang des Gespräches. Sie versucht damit vielmehr eine Nähe zum Kunden aufzubauen. Dieses Generieren von Nähe kommt dem Kommerz zu Gute. Auch wenn im ersten Blick das Switchen ins Hebräische nicht förderlich für den an dieser Stelle endenden Verkaufsakt zu sein scheint, so kann es beim nächsten Marktbesuch des Kunden als ein positive Erinnerung eine neue Verkaufsinteraktion fördern. Das Nachahmen der Sprache des Kunden zeigt eine Wertschätzung seiner Sprache und damit seiner konstruierten Identität. Aber auch das Switchen ins Englische kann durchaus als kommerziell eingestuft werden. Um einen Verkaufsakt zu fördern ist es unerlässlich, dass das Gegenüber die Verkäufer:innen versteht. Das Englische ist für keine:n der Verkäufer:innen eine Sprache, die sie fließend benutzen und in die sie im Alltag normalerweise switchen (sie sind nicht Teil einer bilingualen Sprecher:innengruppe, in der Englisch eine Rolle spielt). Ihre Intention ist durch die gewährleistete Kommunikation das Verkaufsgespräch funktionsfähig zu machen und dient damit dem Kommerz.

In den Fokusgruppeninterviews haben uns die Verkäufer:innen Antworten gegeben, die diese Annahme bekräftigen. In (4) wird auf die Frage geantwortet, warum man versucht, in jemandes Sprache zu sprechen (was die Verkäufer:innen unter ,jemandes Sprache“ verstehen, war ihnen 
überlassen), ob das einen kommerziellen Nutzen bringt (5) und ob nur einige wenige Wörter dafür ausreichend sind (6).

(4)
01 V_OB_01:
Um sich gegenseitig besser zu verstehen.
02 V_KA_03:
03 V_KA_01:
Aber auch um der Nähe/Innigkeit zu erzeugen.
Wegen der Nähe/Innigkeit.
04 V_KA_01: <Auf die Frage, ob es auch deswegen sein kein, dass man zeigen will, das man
05
Teil dieser ,Multikulti“"-Atmosphäre ist> Ja, das ist es natürlich auch.
06 V_KA_03: Ich finde, jemand der keine Nähe zeigt/nicht innig ist, macht sich auch keine
Mühe mit anderen Wörtern <anderssprachigen>.

01 V_KA_01:

Natürlich, je schöner und wärmer eine Atmosphäre ist, desto mehr

03 V_BR_01:

Kund:innen kommen.

04

$<<$ Türkisches Sprichwort> Eine Sprache macht einen Menschen>, je mehr Sprachen du kannst, desto mehr multikulti bist du.

01 mehrere V: Ja.

02 V_OG_01: So ist es.

03 V_BR_01: Mehrsprachigkeit bringt auf jeden Fall einen Nutzen beim Verkauf.

In (4:01) wird das gegenseitige Verständnis, also die oben beschriebene kommunikative Motivation und in (4:02), (4:03) und (4:06) die erzeugte Innigkeit bzw. Nähe betont, was wir aus dem türkischen samimiyet übersetzt haben. Diese Nähe führe zu einer „wärmeren“ Atmosphäre und damit verbunden zur Steigerung des Kommerzes (5:01-02). Interessanterweise wird noch ein Faktor betont, auf den bei der Interpretation von (3) nicht eingegangen wurde. Die Verkäufer:innen sprechen davon, eine „mehrsprachige MultikultiAtmosphäre“ zu erzeugen und sich als ein Teil dieser Atmosphäre zu zeigen (4:05, 5:04, 6:03). ${ }^{7}$ Das heißt, dass nicht nur das Switchen in die Sprache der Kund:innen, sondern auch in andere Sprachen als die, die mit den Zugehörigkeiten der Interaktant:innen in Zusammenhang stehen einem kommerziellen Zweck dienen können. Dieser für die Verkaufinteraktion positive Effekt ist im engen Zusammenhang mit den Spracheinstellungen der Interaktant:innen zu sehen.

Zusammenfassend argumentieren wir dafür, dass die erhobenen Daten insofern ein Forschungsdesiderat beheben, als dass sie offensichtlich machen, wie CS-Phänomene zwischen unerwarteten Sprachen immer wieder neue Sprachkonstellationen ausbilden, die in ihrer Gesamtheit jedoch für das Setting nicht als markiert, sondern als Regelfall zu bewerten sind. Im Zusammenhang mit diesem strukturellen Potenzial des dynamischen Switchens zwischen verschiedenen teils unerwarteten Sprachen zeigt sich die kommerzielle Motivation, die sich an der Schnittstelle von Identitätskonstruktion (Kapitel 4), Sprachkommodifizierung (Kapitel 5) und Kommunikation (Kapitel 6) bewegt, als besonders relevant. Bei der Analyse der Daten finden weitere Ansätze aus der Soziolinguistik Anwendung, insbesondere die Face-Theorie beim Switchen in die Sprache, die mit der angenommenen Zugehörigkeit der Kund:innen einhergeht. Mit Hinblick auf Spracheinstellungen ist insbesondere die Sprachkommodifizierung relevant.

An dieser Stelle ist es angebracht, einige weitere Aspekte klarzustellen, bevor die interagierenden Faktoren des kommerziellen CS analysiert werden. Beispielsweise soll geklärt

\footnotetext{
${ }^{7}$ Der Begriff „Multikulti“ wurde an dieser Stelle aus dem vorigen Verlauf des Interviews aufgegriffen. Die Verkäufer:innen haben sowohl den Begriff „Multikulti“ als auch „Kultimulti“ mehrmals auch in vorigen Gesprächen erwähnt.
} 
werden, was unter einem zusammenhängenden Verkaufsgespräch zu verstehen ist und welche weiteren Interaktionstypen in den Marktaufnahmen vorzufinden sind. Wie sich ein Interaktionstyp kategorisieren lässt, ist für ein Marktsetting wahrscheinlich etwas simpler als für andere Settings. Gespräche aus den audiovisuellen Aufnahmen, die keine Verkaufsinteraktion abbilden, kategorisieren wir als Privatgespräche. Diese finden meist zwischen Verkäufer:innen statt, die an dem gleichen Stand arbeiten. Es können aber auch Verkäufer:innen anderer Stände involviert sein oder auch Besucher:innen des Marktes, die die Verkäufer:innen aus vorigen Interaktionen innerhalb oder außerhalb des Marktes kennen und das Gespräch daher auf ein Smalltalk abzielt und nicht auf den Verkauf. Unterschieden werden solche Interaktionen von Verkaufsgesprächen oder vom Marktschreien, in denen es um den Erwerb bzw. Verkauf oder die Bewerbung eines Produktes geht. In den audiovisuellen Aufnahmen der Verkaufsinteraktionen werden dabei meist die Privatgespräche von Verkäufer:innen durch Verkaufsgespräche unterbrochen. Gespräche können sich aber auch überlagern, indem Verkäufer:innen mehrere Kund:innen gleichzeitig bedienen.

Einen guten Anhaltspunkt bei der Klassifizierung von Interaktionstypen bieten Werkzeuge der interaktionalen Linguistik, auf deren Grundlagen die Analyse des CS auf dem Markt auch beruht. Die interaktionale Linguistik interessiert sich für jede Art interaktionaler Sprachverwendung, also für Sprachverwendung, bei der ein sequenziell strukturierter, kollaborativer und situationsgebundener Bedeutungs- und Strukturaufbau die Grundlage bildet (Imo/Lanwer 2019). Von diesem Blickwinkel betrachtet bildet in unserem Falle der Markt die Situation, in der Verkäufer:innen und Kund:innen Rollen als Interaktionspartner:innen annehmen: V und $\mathrm{K}$ stehen in dieser Arbeit für diese interaktionalen Rollen. V und $\mathrm{K}$ führen kollaborativ Gespräche in Form von sequenziellen und strukturierten Verkaufsgesprächen oder monodirektional in Form von Marktschreien. Das soll nicht heißen, dass alle CS-Phänomene, die in den Interaktionen zwischen $\mathrm{V}$ und $\mathrm{K}$ auftreten, direkt als kommerziell eingestuft werden können, aber kommerzielles CS kann nur einer Interaktion zugeschrieben werden, in der diese beiden Rollen vertreten sind. Das Fundament des kommerziellen CS wird also durch das Setting des Marktes und die Rollen V und K gelegt. Damit sind Privatgespräche mit CS zunächst davon ausgeschlossen, als kommerziell bewertet zu werden. Es gibt aber auch durchaus Fälle, bei denen ein:e Verkäufer:in die Rolle K annimmt und an einem anderen Stand etwas kauft. Diese Fälle zeigen, dass interaktionale Rollen nicht immer synchron zum Verkäuferstatus auf dem Markt verlaufen müssen.

Einen interessanten Einwand würde die Frage zur Folge haben, ob CS-Phänomene in einem Verkaufsgespräch immer kommerziell motiviert sind. Tatsächlich möchten wir nicht dafür argumentieren, dass dies immer zwangsläufig der Fall ist. Der soziolinguistische Hintergrund der Verkäufer:innen spielt hier eine wichtige Rolle. Insbesondere haben wir Beispiele mit einem Switch ins Arabische nur dann als kommerzielles CS-Beispiel angeführt, wenn es von Verkäufer:innen stammt, die Arabisch nicht fließend beherrschen und damit nicht in die arabisch-deutsche bilinguale Sprecher:innengruppe gehören. Ähnlich wurden Beispiele mit Switches ins Türkische nur dann gewertet, wenn sie von Verkäufer:innen stammen, die nicht zur deutsch-türkischen bilingualen Sprecher:innengruppe gehören. Andernfalls wäre eine klare Abgrenzung einer kommerziellen Motivation von dem, was oben als ,mixed code as default“ angeführt wurde und auf die Alltagssprache bilingualer Sprecher:innengruppen referiert, nicht 
immer möglich. Anders verhält es sich beim Marktschreien. Diese Art der Interaktion kann sich zwar an bestimmte Kund:innen richten, hat aber auch immer einen öffentlichen Bezug, der sich an alle Passant:innen richtet. Die Verkäufer:innen sind zwar in einer Interaktion mit den Kund:innen, aber es handelt sich nicht um ein Gespräch in dem Sinne, dass zwei Personen miteinander sprechen. Vielmehr ist die Interaktion monodirektional. Dadurch tritt der Aspekt der bilingualen Sprecher:innengruppe weitesgehend in den Hintergrund.

\section{Daten und Methoden}

Im Forschungsprojekt, in dem der Fokus auf der soziolinguistischen Beschaffenheit und Ethnografie des Maybachufer-Marktes in Berlin-Neukölln liegt, wurden Daten unter Berücksichtigung verschiedener Methoden erhoben, die zum Zweck haben, die hohe Diversität auf dem Markt multiperspektivisch zugänglich zu machen. Unter anderem wurden folgende Methoden bzw. Datensätze für die vorliegende Arbeit verwendet:

- audiovisuelle Aufnahmen der Verkaufsinteraktionen: Nicht gelenkte, spontane Sprechdaten von drei Ständen mit deutsch-türkisch und einem Stand mit deutsch-arabisch bilingualen Verkäufer:innen. Die CS-Beispiele in der vorliegenden Studie stammen aus diesen Aufnahmen.

- soziolinguistische Interviews mit Verkäufer:innen: Fragen zu ihren biografischen und sprachlichen Hintergründen. Details folgen in Kapitel 3.1.1.

- Kurzinterviews mit den Marktbesucher:innen: Interviews mit 224 Kund:innen nach ihren Einkäufen. Fragen zu ihrem sprachlichen Hintergrund, ihrer Beziehung zum Markt und während des Einkaufens aufgefallenen Phänomenen. Details folgen in Kapitel 3.1.2.

- Fokusgruppeninterviews mit den Verkäufer:innen: Interviews in einer lockeren Atmosphäre mit Tee und Snacks, die nach den audiovisuellen Aufnahmen der Verkaufsinteraktionen durchgeführt wurden. So gab es nach der ersten Analyse der Verkaufsgespräche die Gelegenheit, weitere Unklarheiten oder Forschungsfragen zu klären.

\subsection{Teilnehmer:innen}

Die Informationen über die Teilnehmer:innen stammen aus den soziolinguistischen Interviews mit den Verkäufer:innen und aus den Kurzinterviews mit den Marktbesucher:innen. Während der Aufnahmetage wurden weitere Verkäufer:innen vor Ort zu ihrem sprachlichen Hintergrund befragt.

\subsubsection{Verkäufer:innen}

Verkäufer:innen von kooperierenden Ständen haben an soziolinguistischen Interviews teilgenommen. In diesen Interviews wurden sie über ihre persönliche und linguistische Biografie, ihr linguistisches Repertoire, Spracheinstellungen zu bestimmten Sprachen, die sie sprechen und nicht sprechen, besonders, die auf dem Markt gesprochen werden und allgemein über Mehrsprachigkeit und die Besonderheiten der Sprache auf dem Markt befragt. Die Interviews wurden auf Türkisch und/oder Deutsch durchgeführt. Verkäufer:innen, die nicht an diesen Interviews teilgenommen haben, wurden während der Aufnahmetage auf dem Markt zu ihrem sprachlichen Hintergrund befragt. 
$\mathrm{Zu}$ den Ständen gehören ein Obst- und Gemüsestand (OG); ein Textilien- und Haushaltsutensilienstand (TX); ein Kaffeestand (KA); und ein Brot- und Gebäckstand (BR). Außerdem war eine Verkäuferin von einem Schmuckstand (SC) oft an den Ständen BR und KA und wurde auch zu ihrem sprachlichen Hintergrund befragt. Ein weiterer Obst- und Gemüsestand (GO) ist in den Aufnahmen von TX zu hören, besonders das Marktschreien dieses Standes wurde später in die Analyse involviert. In (7) sind Informationen zum sprachlichen Hintergrund von 14 dieser Verkäufer:innen aufgeführt, deren Daten in der vorliegenden Arbeit verwendet wurden. Die Namen wurden durch Siglen anonymisiert, wobei V für Verkäufer:in steht, gefolgt vom Kürzel für den Stand und einer individuellen Nummer. ${ }^{8}$

$\begin{array}{llll}\text { V_OG_01: } & \text { L1: Türkisch } & \text { L2: Deutsch } & \text { L3: Englisch (nicht fließend) } \\ \text { V_OG_02: } & \text { L1: Türkisch/Deutsch } & & \\ \text { V_OG_03 } & \text { L1: Türkisch/Deutsch } & & \text { L3: Englisch (nicht fließend) } \\ \text { V_TX_01 } & \text { L1: Arabisch } & \text { L2: Deutsch } & \text { L3: Englisch (nicht fließend) } \\ \text { V_TX_02 } & \text { L1: Arabisch } & \text { L2: Hebräisch, dann Deutsch } \\ \text { V_TX_03 } & \text { L1: Arabisch/Deutsch } & & \text { L3: Englisch (nicht fließend) } \\ \text { V_KA_01 } & \text { L1: Türkisch } & \text { L2: Deutsch } & \\ \text { V_KA_02 } & \text { L1: Türkisch } & \text { L2: Deutsch } & \\ \text { V_KA_03 } & \text { L1: Türkisch/Deutsch } & & \text { L3: Englisch (nicht fließend) } \\ \text { V_BR_01 } & \text { L1: Türkisch } & \text { L2: Deutsch } & \\ \text { V_BR_02 } & \text { L1: Türkisch } & \text { L2: Deutsch } & \\ \text { V_SC_01 } & \text { L1: Türkisch } & \text { L2: Deutsch } & \\ \text { V_GO_01 } & \text { L1: Türkisch/Kurdisch } & \text { L2: Deutsch } & \\ \text { V_GO_02 } & \text { L1: Arabisch/Türkisch? L2: Deutsch }{ }^{-} & \end{array}$

\subsubsection{Kund:innen bzw. Besucher:innen des Marktes}

Die Kurzinterviews mit den Marktbesucher:innen erzeugen ein generelles linguistisches Bild des Marktes zum Zeitraum der audiovisuellen Aufnahmen. 224 Besucher:innen auf dem Markt wurden während der Aufnahmetage zu ihrer persönlichen und sprachlichen Biografie sowie zu ihrer Beziehung zum Markt befragt. Bei dem größten Anteil der Befragten handelte es sich um Kund:innen an den kooperierenden Ständen. Die Alterspanne der Befragten bewegt sich zwischen 16 und 86 Jahren. Es wurden 45 verschiedene L1-Sprachen und 18 verschiedene L2/L3Sprachen angegeben. In den audiovisuellen Aufnahmen, aus denen die CS-Beispiele entnommen sind, konnten wir den Gebrauch zwölf verschiedener Sprachen feststellen. Tabelle 1 zeigt wie viele der 224 interviewten Marktbesucher:innen diese zwölf Sprachen als eine ihrer gesprochenen Sprachen angegeben haben. Beispielsweise haben 75 von 224 Befragten das

\footnotetext{
${ }^{8}$ L1 steht für Erstsprache. L2 steht für eine später erworbene Sprache, die man in einem Land erworben hat, in dem sie die Hauptverkehrssprache ist. L3 steht für eine später erworbene Sprache, die man nicht in einem Land erworben hat, in dem sie eine Hauptverkehrssprache ist.

${ }^{9}$ Alle Verkäufer:innen, bei denen Englisch als L3 angegeben wurde, haben diese Sprache im schulischen Unterricht gelernt. Sie gaben alle an, dass sie die Sprache nicht fließend sprechen können.

${ }^{10}$ Bei V_GO_02 ist nicht sicher, ob Türkisch eine L1 ist oder später erworben wurde.
} 
Deutsche als L1 und 110 als L2/L3 angegeben. Die Sprachen sind nach der Höchstanzahl der L1-Sprecher:innen sortiert, was durch einen Pfeil gekennzeichnet ist: ${ }^{11}$

\begin{tabular}{|l|l|l|}
\hline \multicolumn{1}{|c|}{ Sprache } & \multicolumn{1}{c|}{ L1 $\downarrow$} & \multicolumn{1}{c|}{ L2/L3 } \\
\hline Deutsch & 75 & 110 \\
\hline Türkisch & 34 & 15 \\
\hline Arabisch & 32 & 5 \\
\hline Englisch & 19 & 119 \\
\hline Italienisch & 9 & 4 \\
\hline Französisch & 8 & 29 \\
\hline Kurmandschi & 8 & 4 \\
\hline Spanisch & 7 & 14 \\
\hline Hebräisch & 3 & 1 \\
\hline Persisch & 2 & 2 \\
\hline Russisch & 1 & 12 \\
\hline Japanisch & 1 & 0 \\
\hline
\end{tabular}

Tabelle 1: Sprachen der Marktbesucher:innen

Nach diesem Überblick über das sprachliche Gesamtbild des Marktes folgen nun Beispiele für kommerzielles CS, das unter dem Einfluss von drei Faktoren konstituiert wird: Identitätskonstruktion (Kapitel 4), Sprachkommodifizierung (Kapitel 5) und Kommunikation (Kapitel 6).

\section{Kommerzielles CS und Identitätskonstruktion}

Die Identitätskonstruktion tritt als ein wichtiger Faktor bei der Konstituierung des kommerziellen CS auf. In (8) werden Ausschnitte aus dem Fokusgruppeninterview in (1) wiedergegeben, wo Verkäufer:innen auf die Frage antworten, welche Sprachen sie auf dem Markt benutzen.

$\begin{array}{ll}\text { (1:01) V_KA_03: } & \text { Wir sagen Sachen auch in anderen Sprachen. } \\ \text { (1:05) V_OG_03: } & \text { Arabisch, Englisch, Deutsch. }\end{array}$

In (1:01) spricht V_KA_03 beispielsweise von ,anderen Sprachen“ und in (1:05) zählt V_OG_03 auch das Deutsche zu diesen ,,anderen Sprachen“, obwohl er diese Sprache fließend sprechen kann (siehe (7)). Der Verkäufer nimmt damit eine soziale Positionierung ein. Die deutsche Sprache wird nicht als ,eigene Sprache“ eingeschätzt, sondern als Sprache der anderen. „Positionality“ gehört nach Buchholz und Hall (2005) neben „emergence“, ,indexicality“, „partialness“ und „relationality“ zu den Prinzipien der Identitätstheorie. Diese fünf Prinzipien der Identität filtern sie aus Ansätzen verschiedener wissenschaftlicher Disziplinen, wie der

\footnotetext{
${ }^{11} \mathrm{Da}$ die restlichen Sprachen, die in den Kurzinterviews mit den Marktbesucher:innen genannt wurden, in den Verkaufsinteraktionen nicht gebraucht werden, sind sie für unsere Analyse nicht weiter relevant und werden in der Tabelle 1 nicht aufgeführt. Für Interessent:innen möchten wir die Sprachen an dieser Stelle vollständig auflisten: Albanisch, Arabisch, Bosnisch, Chinesisch, Dänisch, Deutsch, Englisch, Esan, Französisch, eine ghanaische Sprache (nicht weiter spezifiziert), Griechisch, Hebräisch, Hindi, Italienisch, Jamaikanisch, Japanisch, Katalanisch, Koreanisch, Kroatisch, Kurmandschi, Luxemburgisch, Mongolisch, Mòoré, Niederländisch, Panjabi, Persisch, Philippinisch, Polnisch, Portugiesisch, Rumänisch, Russisch, Schwedisch, Schweizerdeutsch, Serbisch, Slowenisch, Spanisch, Türkisch, Ukrainisch, Ungarisch, Urdu, Vietnamesisch, Wolof, Yoruba, Zaza und der schwäbische Dialekt wurden als L1 und teilweise auch als L2/L3 genannt. Außerdem wurden auch Ga, Indonesisch, Irisch, Norwegisch, Tschechisch und Twi als L2/L3 angegeben.
} 
Soziolinguistik, der linguistischen Anthropologie und der sozialen Psychologie, heraus. Die Prinzipien beleuchten den Begriff der Identität aus verschiedenen Perspektiven, sind jedoch sehr stark miteinander verbunden.

Soziologische, anthropologische und linguistische Studien definieren Sprache als eine soziale Praxis (cf. Labov 1963; Trudgill 1972; Bourdieu 1977; Johnstone/Kiesling 2008). Demzufolge können die sprachlichen Praktiken nur dadurch analysiert und verstanden werden, wenn die realisierenden Akteur:innen und ihre soziale und kulturelle Umgebung auch miteinbezogen werden. Das positionality-Prinzip besagt, dass Sprecher:innen durch ihre Haltung in der Interaktion ihre Zugehörigkeit sowohl zu größeren demografischen Kategorien (beispielsweise definiert durch Alter, Geschlecht, Nation) als auch zu lokalen, ethnografisch begründeten Gruppen und interaktionsspezifischen teilnehmenden Rollen reflektieren (,,positionality principle“, cf. Bucholtz/Hall 2005: 591f.). Auch in den Marktinteraktionen werden unterschiedliche Positionierungen gegenüber anderen anhand des Gebrauchs unterschiedlicher Ressourcen indiziert. Dazu zählen sowohl linguistische Indexe wie Sprachwahl und CS oder grammatische Strukturen, als auch Stellungnahmen im Gespräch je nach Ansprechpartner:innen und Kontext der Interaktion.

Das emergence-Prinzip besagt, dass die Interaktion der Entstehungsort der Identität ist, das heißt, dass diese erst durch die Interaktion konstruiert wird, aus ihr hervorgeht (,emergence principle“, Bucholtz/Hall 2005: 588). Auf dem Markt entstehen die Identitäten unter anderem bei einzelnen Verkaufsbegegnungen bzw. -interaktionen, die durch Teilnehmer:innen mit den Rollen V und K charakterisiert werden. Solche Interaktionen schließen nicht nur den bloßen Verkaufsakt ein, sondern auch Smalltalks, in denen weitere Identitäten (nach den oben genannten Zugehörigkeiten sowie nach den unterschiedlichen Haltungen, die im Laufe der Interaktion eingenommen werden können) konstruiert werden (Duman 2021). Aus diesem Grund ist es essenziell, die interaktionale Linguistik und die linguistische Ethnographie in die Analyse einfließen zu lassen.

Das indexicality-Prinzip besagt, dass sprachliche Praktiken nicht isoliert betrachtet werden können, da sie eng mit dem Kontext der Interaktion in Zusammenhang stehen. Aus der Interaktion hervorgehend können verschiedene sprachliche Praktiken auf lexikalischer, grammatikalischer, phonetischer, stilistischer Ebene und auf Ebene der Wahl eines Codes, unterschiedliche soziale Bedeutungen tragen, die zu einer Identitätskonstruktion führen können (cf. Eckert 2005, 2008, 2016 zur Indexikalität und sozialen Bedeutung; Blommaert 2007 zur Indexikalität in der Diskursanalyse) (,,indexicality principle“, Bucholtz/Hall 2005: 593). In der Tat gaben die Verkäufer:innen während der Fokusgruppeninterviews an, dass sie die Zugehörigkeit der Kund:innen anhand ihrer Sprache oder an Begrüßungen am Anfang von Verkaufsgesprächen generieren (9:02-03).
01 mehrere V:
Am Aussehen / Am Gesicht des/der Kund:in.
02 V BR 01:
03 V_SC_01:
04 V_KA_01:
Je nachdem welche Sprache der/die Kund:in spricht, musst du ihn/sie ansprechen.
Und von den Begrüßungen <am Anfang $>$ ist es gut erkennbar.
06 V_OG_03:
Also die Russ:innen sind sofort bemerkbar, Araber:innen, beispielsweise aus
Syrien, sind sehr reich und qualitativ mit Markenkleidung.
07 V_KA_01:
Und die Roma sind auch sofort erkennbar, weil sie sich sehr bunt anziehen.
$<$ Bestätigt aus vorigem Gespräch, dass Araberinnen und Türkinnen einen ganz anderen Kopftuchstil haben> Leute mit einem Netzbeutel sind meist Deutsche und Engländer:innen. 


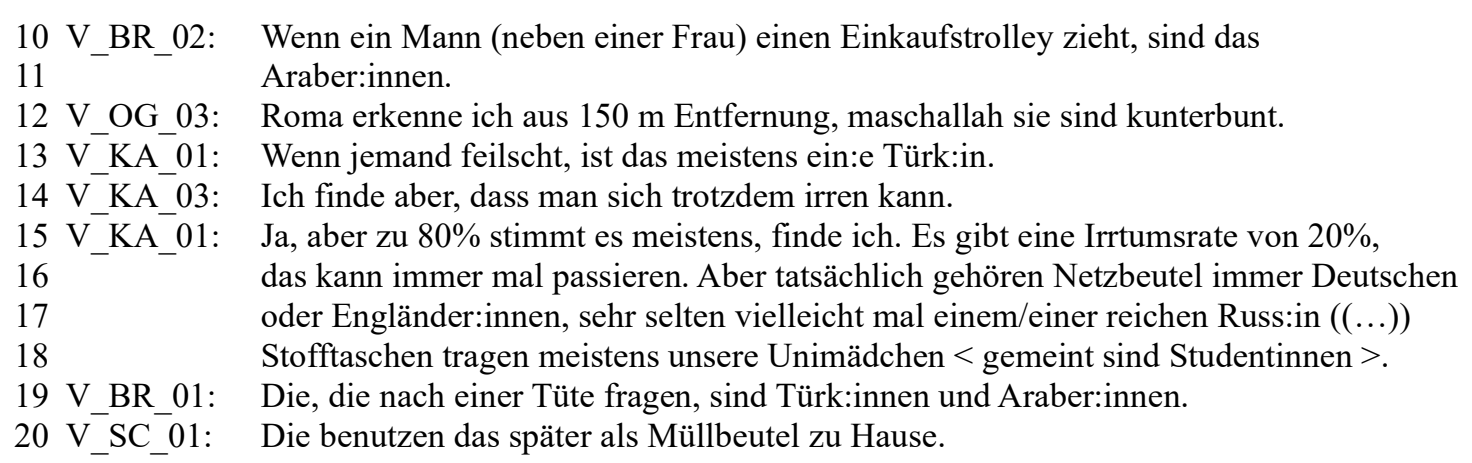

Das partialness-Prinzip besagt, dass neben diesen sprachlichen Ressourcen auch kulturelle Ressourcen in der Interaktion identitätsmarkierend sein können. Sie können zum Teil absichtlich und zum Teil habituell zur Konstruktion der Identität verwendet werden (,partialness principle“, Bucholz/Hall 2005: 605). Die Verkäufer:innen zählen Kleidungsstil (9:04-07, 12), Art der Trageutensilien (9:08-10, 16-18) und soziale Verhaltensweisen $(9: 10-11,13,19-20)$ als kulturelle Ressourcen zur Identitätskonstruktion auf. Aber auch das körperliche Erscheinungsbild und besonders das Gesicht der Kund:innen ist ein wichtiger Indikator für die Zuordnung (9:01).

Allerdings sind die Identifikation und die Zu- bzw. Zusammengehörigkeit (cf. Pfaff-Czarnecka 2011) soziale Prozesse und werden daher immer im Zusammenhang mit anderen sozialen Kategorien und interaktionalen Rollen bestimmt. I. e. die Identität wird immer intersubjektiv und nach verschiedenen Verhältnissen (z. B. Gemeinsamkeit vs. Unterschied oder Echtheit vs. Denaturalisation) konstruiert, und dies macht die Identität relational, was auch das ,relationality“Prinzip besagt. (Bucholtz/Hall 2005: 598).

Zusammenfassend lässt sich sagen, dass in den kommunikativen Situationen des Marktes die Identität durch verschiedene linguistische und im weitesten Sinne semiotische Ressourcen als das Produkt der Interaktion auftaucht, je nach weiteren interaktionalen Haltungen andere Identitätskonstruktionen abruft und den weiteren Verlauf der Interaktion formt. Besonders semiotische Merkmale, wie z. B. das Aussehen oder die Bekleidung, Accessoires wie Tragetaschen in all ihren Formen und linguistische Besonderheiten, wie z. B. ein bestimmter Akzent oder lexikalische Wahl, spielen eine wichtige Rolle bei der angenommenen sozialen oder ethnischen Zugehörigkeit und Zuordnung der sozialen Position. Die getroffenen Entscheidungen hinsichtlich der sprachlichen Ressourcen sind oft das Resultat der Identitätsverhandlungen. Sobald man seine:n Ansprechpartner:in sozial positioniert, wählt man die Ressourcen, die man für die jeweilige Interaktion und konstruierte Identität als relevant betrachtet. So switcht der Verkäufer in Beispiel (10) ins Türkische, weil er von der Kundin eine soziale Zugehörigkeit annimmt, die mit dieser Sprache im Zusammenhang steht. (11) zeigt einen Wechsel ins Arabische. In (12) ist zu sehen, dass Passant:innen, genauer gesagt zwei verschiedene Pärchen, in zwei verschiedenen Sprachen zum Stand eingeladen werden.

\begin{tabular}{|c|c|c|c|c|c|c|}
\hline 01 & V_TX_01 & $<<$ hält Schneeanzug $>$ & acht euro & (.) kostet & acht euro & $\frac{\text { abla.> }}{\text { Schwester }}$ \\
\hline 02 & K_TX_01 & ((nickt und sieht sich J & ans an)) & & & \\
\hline 03 & V_TX_01 & $<<$ zeigt auf Jeans $>$ & $\frac{b u}{\mathrm{DEM}}$ & $\frac{\text { beş euro }}{\text { fünf Euro }}$ & $\frac{a b l a}{\text { Schwester }}(-)$ & fünf eu \\
\hline
\end{tabular}




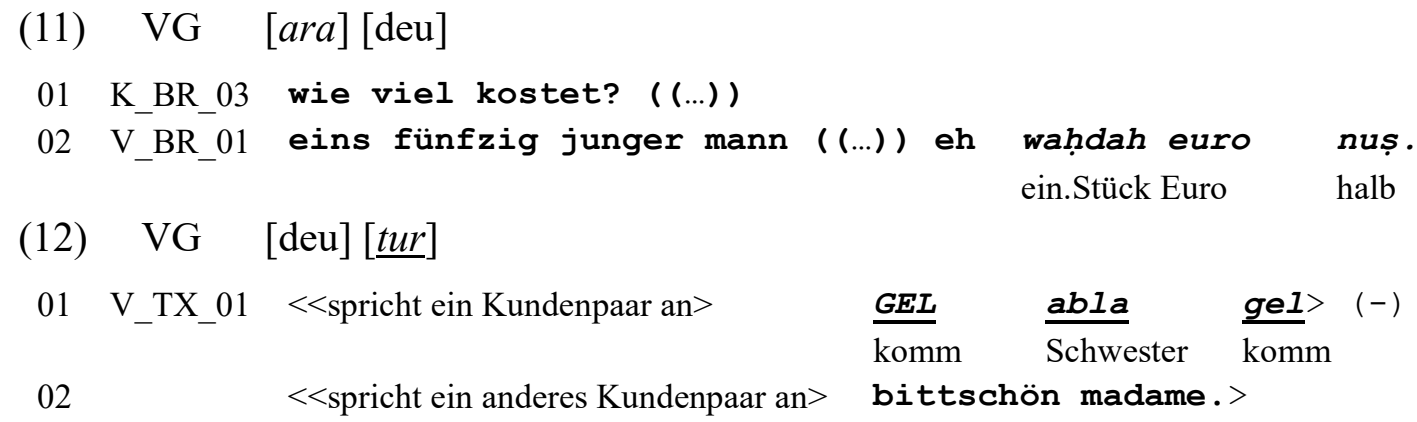

In (13) sind im Zusammenhang der Identitätstheorie weitere interessante Phänomene zu beobachten. Zunächst ist ein Switch in eine iranische Sprache zu sehen, die wir als Kurdisch/Persisch markiert haben. Obwohl die phonetische Struktur dem Kurdischen ähnelt, assoziiert der Verkäufer den Kunden mit einer afghanischen Zugehörigkeit (13:02), die generell mit der persischen Sprache assoziiert wird. Der Kunde akzeptiert die angenommene Zugehörigkeit zu Afghanistan (13:03). Da sich der Wortlaut der Numerale in beiden Sprachen sehr ähnelt und das dem Verkäufer eventuell bewusst ist, beabsichtigt er wahrscheinlich Persisch zu benutzen. Interessanterweise verwendet er im weiteren Verlauf der Interaktion die Anredeform kardaş (= kardeş, türk. dialektal für ,Bruder/Schwester'). Obwohl das nicht die Sprache ist, die mit der Zugehörigkeit des Kunden im Zusammenhang steht, kann dieser Switch auch mit ,Innigkeit“ interpretiert werden. Der Verkäufer adressiert den Kunden in der Sprache seiner eigenen Zugehörigkeit mit einem Begriff, der an sich schon durch seine familiäre Domäne Nähe ausdrückt. ${ }^{12}$

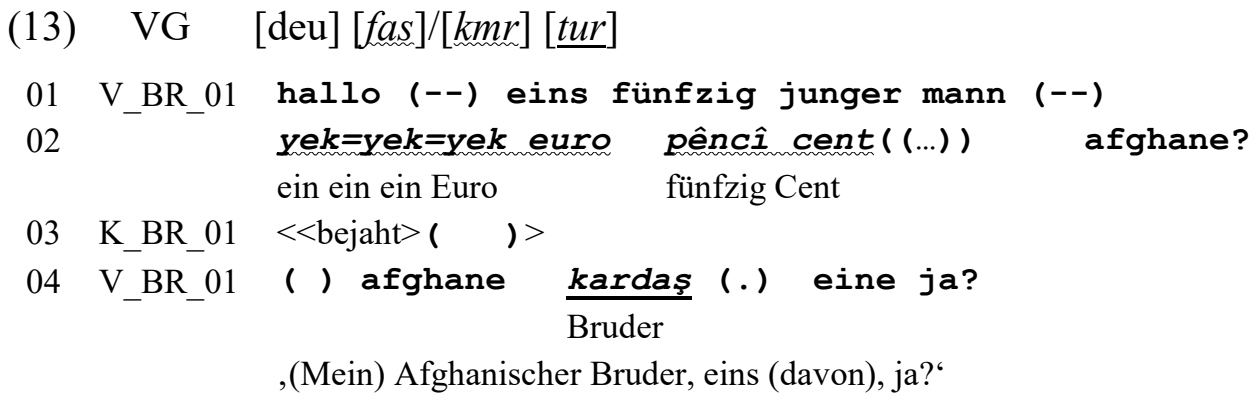

$\mathrm{Ab}$ (9:14) in den Antworten der Verkäufer:innen in den Fokusgruppeninterviews sieht man aber, dass sie sich bei ihrer Annahme und Positionierung durchaus irren können. Im anfänglichen Beispiel (3) hat sich die Verkäuferin V_KA_01 gleich zweimal in ihrer Annahme über eine auf die Zugehörigkeit des Kunden abzielende Sprache geirrt und hat durch den Kunden einen neuen Input bekommen. (14) und (15) zeigen Fälle, wo der Verkäufer keinen neuen Input bekommt. In (14) korrigiert er sich nach dem Bemerken seines Irrtums selbst. Seine Sprache wechselt vom Türkischen ins Arabische. Das deutsche „,bittschön“ bewerten wir hier außerhalb dieser Identitätskonstruktionen. Zum einen, weil Deutsch die allgemeine Verkehrssprache des Marktes ist und zum anderen, weil der Ausdruck „,bitt(e)schön“ sehr frequent in den Marktdaten auftaucht und in einem anderen Zusammenhang analysiert werden kann (in Kapitel 7 wird das Thema kurz angeschnitten). In (15:07) wird er ausdrücklich von der Kundin darauf hingewiesen

\footnotetext{
${ }^{12}$ Es ist gut möglich, dass der Kunde diesen Ausdruck versteht. Eine Evidenz haben wir dafür nicht. Nur aus persönlicher Erfahrung sehen wir eine Tendenz in nicht-türkischen, muslimischen Sprecher:innengruppen, den Begriff „kardeş“ aus dem Türkischen zu kennen.
} 
„abi ich bin nicht türkisch“, woraufhin er ins Arabische switcht, was eine zutreffende zweite Annahme war.

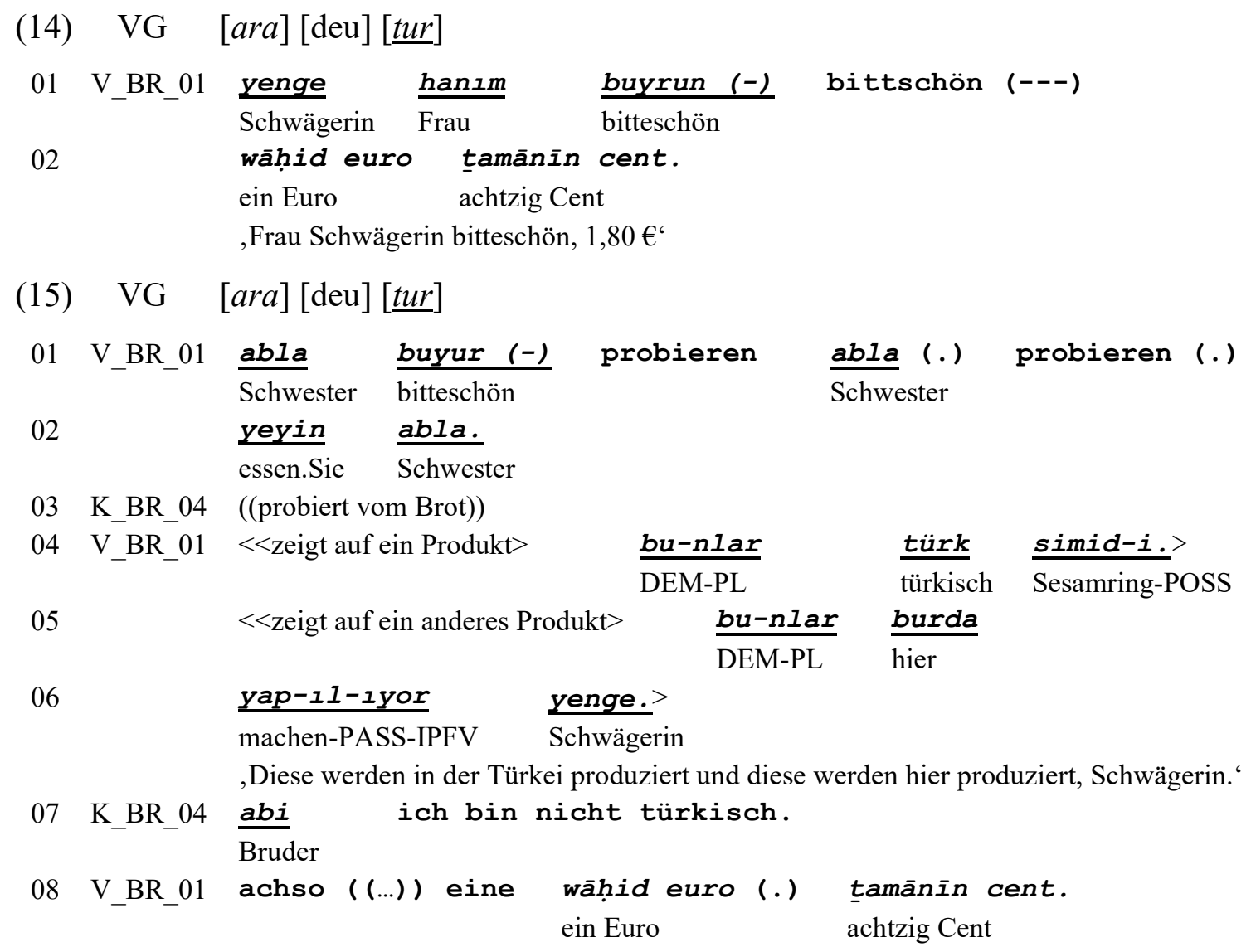

Auch monodirektionale Interaktionen, die sich in Form von Marktschreien zeigen, können kommerzielles CS beinhalten, die auf die soziale Zugehörigkeit der Kund:innen referiert. Verkäufer:innen adressieren dabei mehrsprachig Passant:innen und somit potenzielle Kund:innen. Mit dem Deutschen als stärkste Verkehrssprache des Settings wird einerseits die Allgemeinheit angesprochen, andererseits aber auch besonders Besucher:innen, deren soziale Zugehörigkeit mit dem Deutschen assoziiert wird, da durch das Switchen in andere Sprachen auch das Deutsche hervorgehoben wird. In (16) ist zu sehen, wie das Deutsche mit dem Türkischen kombiniert wird, in (17) mit Russisch und in (18) mit Englisch, Italienisch und Spanisch.

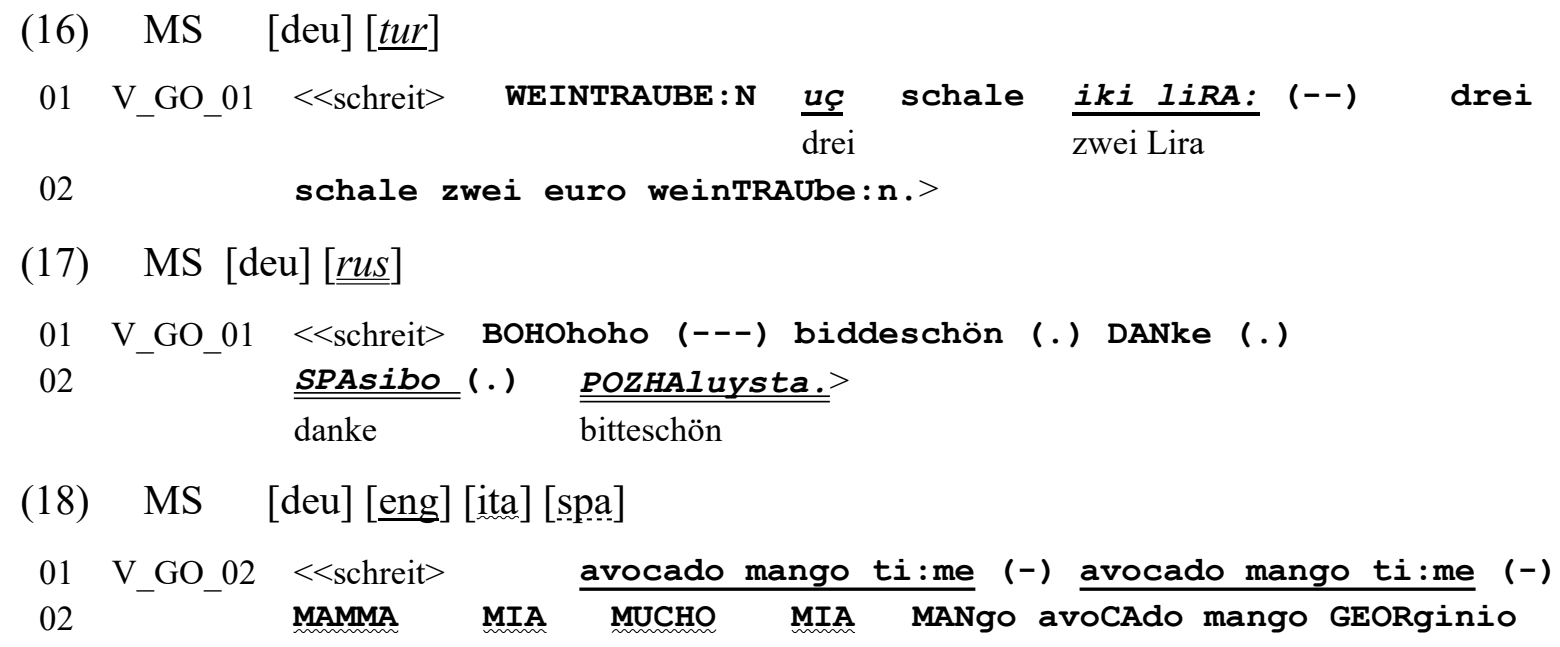


03

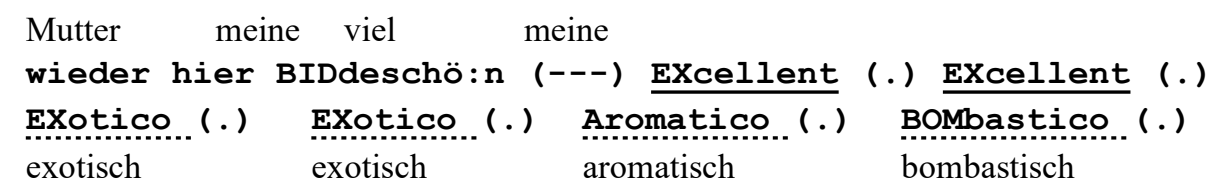

Diese Beispiele können auch aus der Perspektive der Sprachkommodifizierung analysiert werden. Besonders (17) und (18) werden dafür in Kapitel 5 noch einmal herangezogen.

Durch die theoretischen Ansätze zur Identitätskonstruktion wurde nun zwar erklärt, auf welcher Basis sich Verkäufer:innen für eine bestimmte Sprache oder einen bestimmten Ausdruck entscheiden. Nicht geklärt wurde hingegen, warum genau die Benutzung einer auf die soziale $\mathrm{Zu}-$ gehörigkeit abzielenden Sprache bei Kund:innen positiv wahrgenommen wird und dadurch einen Verkaufsakt begünstigen kann. Die Verkäufer:innen selbst sprechen von Nähe/Innigkeit (4). Sie wurden auch gefragt, was sie empfinden, wenn Kund:innen versuchen sie auf Türkisch, also in der Sprache anzusprechen, die mit ihrer Zugehörigkeit assoziiert wird. In den Antworten fiel neben Nähe auch der Begriff der Wertschätzung auf (19).
01 V_OG_01: Das gefällt mir sehr.
02 V_BR_01: Ich mag das sowas von. $((\ldots))$ deren Akzent und wie die die Sätze bilden.
03 V_KA_01: Dass sie uns wertschätzen.
04 V_BR_01: Genau, das stimmt. Und ich fühle mich sehr nah an diese Menschen $((\ldots))$

Um Nähe und diesen Wertschätzungsprozess analysieren zu können eignet sich besonders der Ansatz der Face-Theorie. Nach Goffmann (1967) ist Face ein positiver sozialer Wert, den jemand für sich selbst beansprucht und der durch Aussagen und Verhaltensweisen anderer während einer Kontaktsituation gebildet wird. Die Faces, die von Menschen angenommen werden, sind kontextgebunden und hängen von der Situation, der Kultur der Gesellschaft und von anderen Teilnehmer:innen der Interaktion ab (cf. Redmond 2015). Menschen agieren mit verschiedenen Faces, teilweise gleichzeitig oder ein Face dominiert in verschiedenen Situationen (Tracy 1990). Wir haben also Faces im Kontext einer Interaktion oder in Beziehung mit anderen. Wie mehrmals erwähnt herrscht aus der Perspektive der interaktionalen Linguistik auf dem Markt eine Situation mit einer generellen zweigliedrigen Rollenverteilung: V und K. Im Rahmen des Face-Ansatzes beansprucht jemand auf dem Markt schon allein durch die Annahme der Rolle K ein Face, das von den Interaktionsteilnehmer:innen mit der Rolle V als solches behandelt werden will. V kann dieses Face von $\mathrm{K}$ in ganz unterschiedlichen Formen bewahren. Neben einem höflichen Umgang, Hilfestellung und Beratung zu den angebotenen Produkten, trägt auch die Benutzung von Ausdrücken aus der Sprache der Kund:innen zur Bewahrung und Stärkung dieses Face bei. Ganz grundlegend ist aber, dass sich V bei einem Kaufwunsch von $\mathrm{K}$ in eine Verkaufsinteraktion begibt.

Bevor wir stärker auf die Face-Prozesse in den Verkaufsinteraktionen eingehen, ist es an dieser Stelle hilfreich, sich die Kategorisierung von Face anzusehen. Brown/Levinson (1987) betrachten Face dichotom: Das positive Face ist der Wunsch, in gewissen Aspekten anerkannt zu werden. Beispielsweise besteht das Face von Lehrer:innen darin, als authentische und respektable Lehrperson anerkannt und behandelt zu werden. Das negative Face ist der Wunsch, ungehindert zu sein und dass andere sich nicht einmischen (cf. Brown/Levinson 1987). Beim kommerziellen CS auf dem Markt kommt besonders dem positiven Face eine zentrale Bedeutung zu. 
Kommunikationswissenschaftler Lim/Bowers (1991) benutzen andere Begriffe und unterteilen das, was Brown/Levinson (1987) als positives Face auffassen würden, noch einmal in Competence-Face und Fellowship-Face. Fellowship-Face ist der Wunsch, in eine Gruppe inkludiert zu werden bzw. als Mitglied einer Gruppe wertgeschätzt zu werden. Competence-Face ist der Wunsch, dass die eigenen Fähigkeiten wertgeschätzt oder respektiert werden. Diese beiden Arten des Face müssen nicht zwingend parallel verlaufen. Eine Gruppe kann akzeptieren, dass jemand gewisse Fähigkeiten hat, aber diese Person trotzdem aus ihrer Gruppe ausschließen. Wir benutzen diese beiden Begriffe, um Praktiken auf dem Markt zu beschreiben, die mit dem Aufbau, der Erhaltung/Bewahrung oder der Verletzung des Face zu tun haben und unter FaceArbeit zusammengefasst werden (cf. Goffmann 1955; Domenici/Littlejohn 2006). Zu dieser Face-Arbeit gehört nach Goffman (1955) und O'Keefe/Stephard (1987) beides, die Bewahrung des eigenen Face und die Unterstützung, dass Interaktionspartner:innen ihr Face bewahren können.

Wir haben festgehalten, dass es ganz grundlegend ist, dass sich ein:e Verkäufer:in bei Wunsch des/der Kund:in in eine Verkaufsinteraktion begibt. Damit wird das Fellowship-Face des/der Kund:in bewahrt, welches er/sie als Mitglied des Marktes in der Rolle K hat. Durch den Switch in eine Sprache, die mit der sozialen Zugehörigkeit der Kund:innen in Zusammenhang steht, wird dieses Fellowship-Face der Kund:innen weiter gestärkt. Kund:innen, die in ein Verkaufsgespräch involviert sind, oder Passant:innen, die an den Ständen vorbeilaufen und hören, dass in einer Sprache geschrien wird, zu der sie eine Zugehörigkeit haben, fühlen sich zusätzlich als Marktmitglied inkludiert. Außerdem wird neben dem Fellowship-Face in Bezug auf die Marktsituation, noch das Fellowship-Face als Teil einer Sprecher:innengruppe gestärkt, indem ihre Sprache als so wertvoll gesehen wird, dass sie gelernt oder gesprochen wird, was wir weiter oben als Wertschätzungsprozess benannt haben (siehe (19)).

Nicht nur das Fellowship-Face der Kund:innen wird gestärkt, die Verkäufer:innen bewahren damit auch ihr eigenes Competence-Face, da sie so ihrer Rolle als V nachkommen können, aber auch, dass sie in einer zusätzlichen Sprache sprechen können. Durch eine positive Reaktion der Kund:innen, beispielsweise ein Anlächeln, Antworten oder im besten Fall den Kauf von Produkten, wird dieses Competence-Face bestätigt und gestärkt. Abbildung 1 zeigt die Zusammenhänge zwischen semiotischem Input für die Identitätskonstruktion/Zuordnung der Kund:innen (gepunktete Linie), dem kommerziellen CS, der als Output daraus folgt (durchgezogene Linie) und den dadurch aktivierten Face-Prozessen (Doppellinien). 


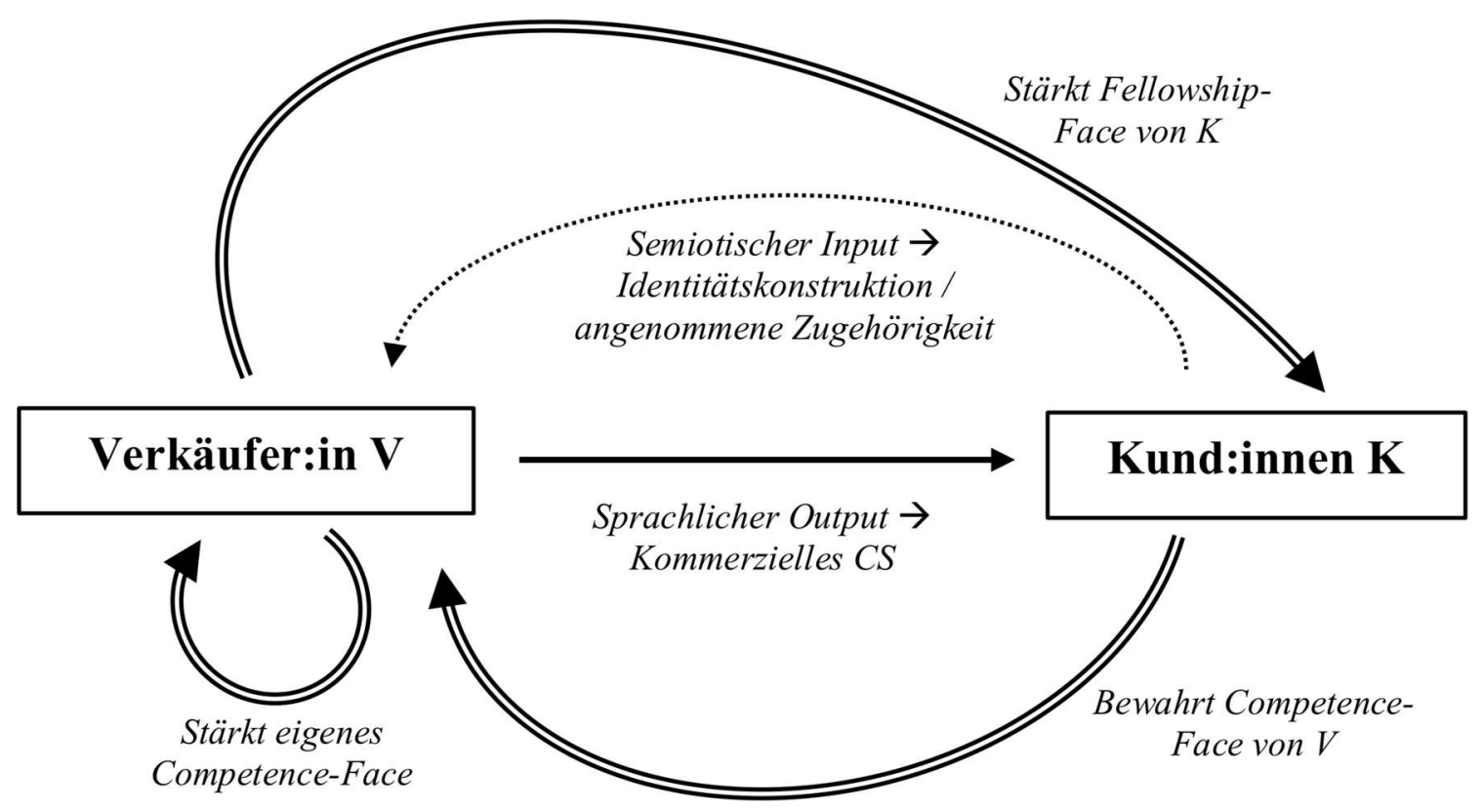

Abbildung 1: Prozesse auf verschiedenen Ebenen beim kommerziellen CS

In der Face-Literatur werden Face-verletzende Praktiken unter den Begriffen „,face-threatening acts“, „to be out of face“ oder ,,shameface“ versammelt (Brown/Levinson 1987; Goffman 1955; Domenici/Littlejohn 2006). Darunter fallen Praktiken, die sich konträr zu den Anforderungen zur Bewahrung des Face verhalten (cf. Brown/Levinson 1987). In den kommerziellen CS-Phänomenen des Marktes geschieht dies meistens ungewollt, denn nicht immer führt die Identitätskonstruktion dazu, eine Sprache zu erschließen, die das Face der Kund:innen stärkt, was in den Beispielen (3), (14) und (15) beobachtbar ist. Dennoch kann allein die Absicht der Verkäufer:innen als positiv bewertet werden. Das sehen wir daran, dass der Kunde in (3) die falsche Sprachwahl mit Humor nimmt, lacht und in guter Stimmung einen neuen sprachlichen Input gibt. Dadurch bewahrt er sowohl sein Face als K als auch das Face des Gegenübers als V. Etwas mit Humor zu nehmen, zu akzeptieren und sich zu berichtigen, zu ignorieren oder zu verleugnen, um Entschuldigung bitten oder die Diskretion sind Strategien unter Interaktionspartner:innen, um sowohl ihr eigenes verletztes Face als auch das des Gegenübers zu behandeln oder wiederaufzubauen (Redmond 2015). Auch in (14) und (15) berichtigt sich der Verkäufer und switcht in eine Sprache, die das Face der Kund:innen auf richtige Weise stärken soll.

\section{$5 \quad$ Kommerzielles CS und Sprachkommodifizierung}

Bei einigen Beispielen aus den Daten ist es nicht möglich, eine direkte Verbindung zu Identitätskonstruktionen zu ziehen. Besonders im Marktschreien werden nicht immer bestimmte Kund:innen adressiert. Verkäufer:innen schreien in die Menschenmenge und versuchen alle potenziellen Kund:innen zu erreichen, die sie hören. Inwieweit dabei das CS auf einer Identitätskonstruktion beruht, ist für einige Beispiele nicht zu erschließen. Die Benutzung von Sprachen wie Türkisch (siehe Beispiel (16)) spricht sicherlich die soziale Zugehörigkeit einiger Passant:innen an, die diese Sprache als L1 sprechen, denn Türkisch ist mit 34 L1-Sprecher:innen unter 224 Befragten die zweitstärkste Sprache auf dem Markt, dicht gefolgt von Arabisch (siehe Tabelle 1 in Kapitel 3). Das oben unter (18) angeführte Beispiel mit dem Italienischen und 
Spanischen bedarf aber einer alternativen Analyse, die den Faktor der Identitätskonstruktion nicht im Vordergrund hält. Denn sowohl das Italienische mit 9 L1-Sprecher:innen, als auch das Spanische mit 7 L1-Sprecher:innen fallen da viel schwächer in der Anzahl der potenziellen Kund:innen aus. Wieso also switchen Verkäufer:innen zu Sprachen, mit denen wahrscheinlich sehr wenige Kund:innen sich in ihrer Zugehörigkeit wertgeschätzt fühlen?

Das Switchen kann durch unterschiedliche Faktoren begründet werden. Ein wichtiger Aspekt sind Spracheinstellungen. In den soziolinguistischen Interviews mit den Verkäufer:innen (siehe Kapitel 3) wollten wir anhand von direkten und indirekten Fragen Einstellungen der Verkäufer:innen gegenüber bestimmten Sprachen erfassen. In (20) ist die Antwort von V_OG_01 auf die Frage „Welche Sprache ist für Sie die schönste oder klingt für Sie am schönsten?“ wiedergegeben.

,Auch wenn ich es nicht spreche, finde ich Spanisch sehr schön. '13

Außerdem haben wir mit Fragen wie „Wenn Sie Sprachen mit Kleidungsstücken vergleichen sollten - welches Kleidungsstück wäre Türkisch, Deutsch, Englisch oder Spanisch für Sie? Welche Farben würden Sie diesen Sprachen geben?“" erzielt, dass die Verkäufer:innen ihre Einstellungen und Gefühle, die sie mit einer bestimmten Sprache assoziieren, besser zum Ausdruck bringen können. In (21) wird exemplarisch die Antwort von V_OG_01 wiedergegeben.

(21) ,Türkisch ist eine Jacke, weil es einen umarmt und warmhält und ist rot, weil das die Farbe der türkischen Flagge ist. Deutsch ist eine Sommerlatsche, du ziehst sie nur an, wenn du sie brauchst und ist violett, weil ich diese Farbe nicht so mag. Englisch ist ein Smoking, weil man das für spezielle Anlässe anzieht und ist weiß, weil es eine saubere und höfliche Sprache ist. Spanisch ist ein T-Shirt, was sowohl über eine Jeans, als auch über eine Stoffhose angezogen werden kann und ist schwarz, weil es etwas unhöflicher klingt [als Englisch]. Trotzdem mag ich diese Sprache. ${ }^{\text {}}{ }^{14}$

Außerdem wurde Deutsch von anderen Verkäufer:innen mit einem „Anzug“ assoziiert, weil es eine ,intelligente/wissenschaftliche“ Sprache sei und mit den Farben rosa und grün, weil Deutschland so grün sei und im Frühling die Blumen blühen würden. Sowie Spanisch wurde auch Englisch und Französisch als „schönste Sprache“ genannt. In Gesprächen mit einigen Kund:innen wurde das Spanische als „leidenschaftlich“ und „warm“ attribuiert. Diese Antworten zeigen, dass Sprachen für gewisse Gefühle stehen oder mit gewissen Elementen assoziiert werden können, die die sprachlichen Praktiken auf dem Markt beeinflussen könnten.

Die Benutzung des Spanischen in Beispiel (18) als schöne und leidenschaftlich kategorisierte Sprache könnte aus dem Aspekt der Spracheinstellungen begründet werden. Sicher spielt es auch eine Rolle, dass man als Deutschsprecher:in und Nicht-Spanisch-Sprecher:in, Wörter wie „exótico“, „,aromático“, „,bombástico“ und „fantástico“ einer Bedeutung zuordnen kann, da es sich hier in Hinblick auf das Deutsche um sogenannte Kognaten handelt, also um Wörter, die sich in ihrer Übersetzung phonetisch überschneiden (exótico - , exotisch', aromático - , aromatisch` etc.). Das könnte bedeuten, dass deutschsprachige Passant:innen die Formen sowohl

\footnotetext{
13 Übersetzung aus dem Türkischen durch die Autor:innen des vorliegenden Beitrags.

14 Übersetzung aus dem Türkischen und Anmerkung in eckigen Klammern durch die Autor:innen des vorliegenden Beitrags.
} 
verstehen, als auch als schön/leidenschaftlich empfinden könnten. Eine weitere Sprache, die in (18:01) sowie in (22) verwendet wird, ist das Englische.

\section{(22) MS [eng]}

\section{V_OG_01 don't wörry be happy ladies.}

Neben den positiven Einstellungen, die das Englische als schön, sauber und höflich kategorisierte Sprache genießt, wird es von vielen Marktbesucher:innen verstanden, auch wenn es sich nicht um Kognaten handelt. 119 von 224 Marktbesucher:innen haben das Englische als L2/3 angegeben (siehe Tabelle 1). Daneben genießt das Englische weltweit ein Prestige als Marketing- und Werbesprache, was sicherlich auch auf dem Markt eine Rolle spielt (cf. Steinbach 1984; Bhatia 1992; Kelly-Holmes 2005; Puntoni et al. 2008; Gerritsen et al. 2010; Cameron 2012). Diesbezüglich argumentiert Puntoni et al. (2008: 1f.): „No area of media production exemplifies the growing importance of the English language better than advertising. Regardless of their cultural heritage and native language, consumers are routinely addressed by large numbers of marketing messages in English.“

19 von 224 Befragten haben das Englische aber als L1 angegeben, weshalb es sicherlich auch aus dem Aspekt der Identitätskonstruktion interpretiert werden kann. Neben den Spracheinstellungen gegenüber einer bestimmten Sprache kann auch die Mehrsprachigkeit selber als ein positives Attribut aufgefasst werden. Das heißt, dass das Marktschreien in vier unterschiedlichen Sprachen in (18) positiv bei den Kund:innen ankommen kann. Die Fokusgruppeninterviews bieten Anhaltspunkte dafür (23).

04 V_KA_01:

05
03 V BR 01:

03 V BR 01:
$<$ Auf die Frage, ob es auch deswegen sein kein, dass man zeigen will, das man Teil dieser „Multikulti“-Atmosphäre ist> Ja, das ist es natürlich auch.

$<<$ Türkisches Sprichwort $>$ Eine Sprache macht einen Menschen $>$, je mehr Sprachen du kannst, desto mehr multikulti bist du.

Mehrsprachigkeit bringt auf jeden Fall einen Nutzen beim Verkauf.

Die Verkäufer:innen sprechen davon, eine „mehrsprachige Multikulti“-Atmosphäre zu erzeugen und sich als ein Teil dieser Atmosphäre zu zeigen. Mehrsprachigkeit selbst kann also auch einem kommerziellen Zweck dienen. Die Benutzung des Russischen in (17) könnte auch im Rahmen dieser „mehrsprachigen Multikulti““-Atmosphäre bewertet werden. Denn von $224 \mathrm{Be}-$ fragten gab nur eine Person Russisch als ihre L1-Sprache an.

Solche Beispiele fallen nicht nur in der Domäne des Marktschreiens auf. In (24) sehen wir in einem Verkaufsgespräch, wie ein Verkäufer den Preis seines Produktes in verschiedenen Sprachen aufzählt und zudem die Mehrsprachigkeit mit ,all sprachen (.) all language“ offen betont.

01 V_TX_01

02 K_TX_06

03 V_TX_01

04

[ara] [deu] [eng] [tur]

faḍdalì $\quad y^{a}$ uhht-ì .

bitteschön oh Schwester-1SG.POSS

$<<$ zeigt auf Produkt und fragt wahrscheinlich nach dem Preis $>(\quad)>$

hamse euro (.) fünf euro(.) five euro (.) beş euro (.)

fünf Euro

fünf Euro

all sprachen (.) all language.

,Fünf Euro in allen Sprachen.

Auch einzelne Sprachen in Verkaufsgesprächen können aus der Perspektive von Spracheinstellungen motiviert werden. Beispiel (25) zeigt, wie eine Einleitung eines 
Verkaufsgesprächs auf Französisch vorgenommen wird, ohne dass es in einem Zusammenhang mit der angenommenen Zugehörigkeit der Kund:innen steht. Denn nach eigenen Angaben des Verkäufers hat er die Kund:innen mit einer deutschen bzw. deutschsprachigen Zugehörigkeit assoziiert. Das Französische wurde in den soziolinguistischen Interviews auch als schöne Sprache eingestuft und wird hier wahrscheinlich aus dieser Spracheinstellung heraus benutzt. Mit seiner Intonation wirkt der Verkäufer schmeichelnd. Aber auch das Switchen ins Deutsche in (26), welches als intelligente Sprache eingestuft wurde, kann aus diesem Blickwinkel gedeutet werden. Denn gerade auf Deutsch wird die Authentizität des Produktes betont.

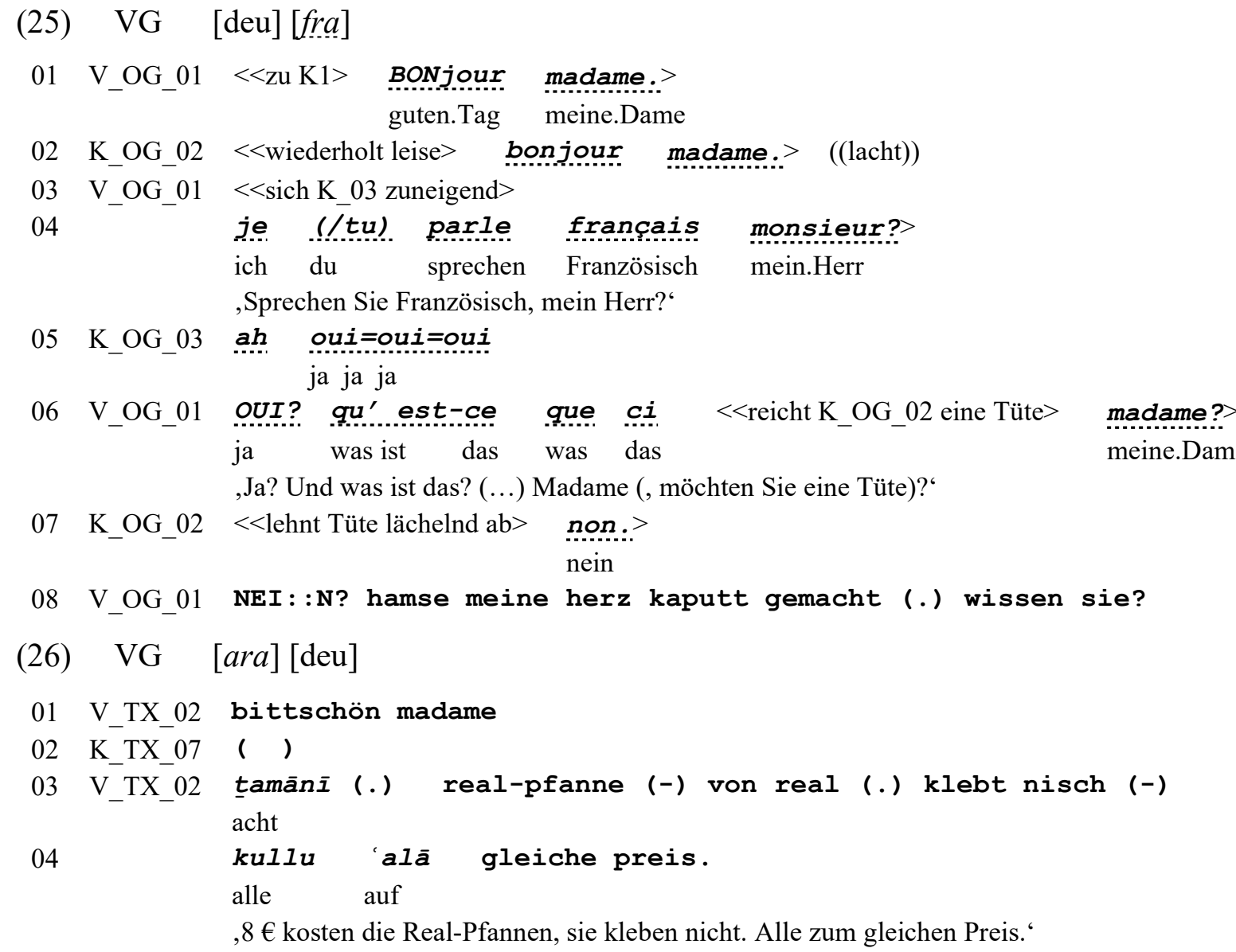

Beispiel (26) ist die einzige Ausnahme zu der in den Präliminarien (Kapitel 2) erwähnten Einschränkung, dass wir nur deutsch-arabische Beispiele aufnehmen, die von türkischsprachigen Verkäuferinnen stammen. Denn sonst wäre die Interpretation einer kommerziellen Motivation anfechtbar, weil es sich nicht von einem ,mixed code as default“ einer bilingualen Sprecher:innengruppe differenzieren ließe. Aber in diesem Beispiel interpretieren wir das Geschehen nicht aus der Perspektive der Identitätskonstruktion, wo das Deutsche oder Arabische die soziale Zugehörigkeit der Kund:innen anspricht, sondern durch Spracheinstellungen gegenüber dem Deutschen, die die Verkäufer:innen teilen.

Die Beispiele in diesem Abschnitt können in einen größeren theoretischen Rahmen eingeordnet werden, der in der Literatur unter Begriffen wie ,linguistic commodification“, ,language commodification“ oder „commodification of language“ aufgeführt wird (cf. Block 2018; Heller 2010; Heller/Duchêne 2016). In dieser Arbeit benutzen wir den Begriff „Sprach- 
kommodifizierung“. Eine der älteren Arbeiten, in der dieses Phänomen angesprochen wird, ist Coulmas (1992). Er beschreibt es wie folgt:

[L]anguages have market value that is the exchange value a certain language has as a commodity, or the index of its appreciation by a relevant group as compared to other languages. The commodity nature of languages manifests itself most clearly in the demand of foreign language learning which can be described as market. The factors determining the market value of a language at a given point in time are various kinds, political, cultural, but above all economic.

(Coulmas 1992: 77f.)

Für unsere Beispiele heißt das, dass einzelnen Sprachen wie Spanisch, Englisch, Französisch und Deutsch oder der Mehrsprachigkeit von Verkäufer:innen ein kommerzieller Mehrwert zugeteilt wird, der auf Spracheinstellungen beruht, die durch verschiedene Faktoren entstanden sind. Heller (2010: 102) erklärt die Sprachkommodifizierung aus einer historischen Perspektive und gibt an, dass sie auf zwei Ebenen erklärt werden muss: Eine Ebene bezieht sich auf Austauschformen, die früher diskursiv als Angelegenheiten der intellektuellen Kompetenz oder des rationalen Denkens behandelt wurden, heute aber als austauschbar für einen materiellen Wert, insbesondere für Geld, betrachtet werden. Mit Austauschformen bezieht sich Heller (2010: 102) beispielsweise auf die Kompetenz standardisierter Sprachnutzung für Berufe. Die andere Ebene bezieht sich auf die Zirkulation von Gütern, die heute, im postkolonialen Zeitalter, mehr denn je vom Einsatz kommunikativer Fähigkeiten und linguistischer Ressourcen abhänge. Im Gegensatz dazu habe früher die physikalische Stärke Priorität bei der Berufsfindung gehabt.

Block (2018) kritisiert den Begriff der, ,Sprachkommodifizierung“ in Hinblick auf zwei Punkte. Zum einen kritisiert er, dass sich die Bedeutungsgebung des Begriffes mit dem Ursprung des Begriffes in Marx (1993 [1858]) nicht vereinbaren lässt. Demnach verläuft die Kommodifizierung in vier Schritten, nämlich der Produktion, der Distribution, dem Austausch und der Konsumption. In der Tat ist in Heller (2010) ein starker historischer Bezug auf Marx, die postkoloniale Zeit und den Kapitalismus vorhanden. Die linguistischen Phänomene in unseren Daten können und möchten wir nicht auf diese Weise historisch einordnen. Es geht uns, wie oben beschrieben, nur um den kommerziellen Mehrwert einzelner Sprachen, der durch einen Einfluss von Spracheinstellungen zugeteilt wird.

Ein weiterer Kritikpunkt Blocks (2018) richtet sich an einige Beispiele, die Heller (2010) für Sprachkommodifizierung nennt. Er findet, dass der Vorteil des Beherrschens einer bestimmten sprachlichen Varietät, beispielsweise bei der Berufsfindung für ein Callcenter, nicht als Kommodifizierung von Sprache, sondern als self-presentation und branding analysiert werden kann. Er begründet seine Ansicht damit, dass die Benutzung einer bestimmten Varietät in diesem Beruf nicht trennbar ist von der Fähigkeit, Menschen anzusprechen, Smalltalk zu führen, Telefonate anzunehmen, feste sprachliche Vorformulierungen abzulesen und zu generieren etc. Block (2018: 133) argumentiert aber auch: „All of this is very different from saying that language is commodified unless we mean bundles of features of language converted into some notion of skill separable from other actions constituting labour power."

Wir denken, dass gerade in unseren Beispielen diese Trennung von Bündeln sprachlicher Merkmale sehr gut funktioniert. Myers-Scotton (1993) schreibt, dass es in jeder multilingualen 
Community eine markierte und unmarkierte Art des Sprachwechsels gibt, also dass die benutzte Sprache erwartet oder unerwartet in Hinblick auf einen bestimmten Kontext ist. Die CS-Phänomene in den obigen Beispielen bilden jeweils markierte Switches in ihren Kontexten, sie tauchen im Vergleich zu Beispielen, die auf einer Identitätskonstruktion beruhen, unerwartet auf und teasen ${ }^{15}$ damit Marktbesucher:innen. So sind sie nicht nur auf syntaktischer Ebene, sondern auch durch ihre Markiertheit in der Motivationsebene trennbar von anderen Aktionen.

Vergleichbare Arbeiten zum kommerziellen Gebrauch von CS liefern Kelly-Holmes (2000, 2005) und Bishop et al. (2005) in der Forschung zu Marketing und Advertising. Im Unterschied zur vorliegenden Studie beschäftigen sie sich mit Werbung aus Massenmedien. Obwohl sie nicht von einer Kommodifizierung sprechen, ordnet sie Heller (2010) dieser Domäne zu.

\section{$6 \quad$ Kommerzielles CS und Kommunikation}

Der dritte Faktor, dem bei der Konstituierung einer kommerziellen Motivation hinter CS eine wichtige Rolle zukommt, ist die Kommunikation bzw. die Gewährleistung der Kommunikation durch eine Sprache oder einen Code, den das Gegenüber versteht. Die Absicht dahinter ist das Verkaufsgespräch zu ermöglichen oder zu vereinfachen. Somit ist dieser Faktor mit einer kommerziellen Motivation verbunden.

Kund:innen auf dem Markt sind nicht immer in der Lage auf Deutsch zu kommunizieren oder sie bevorzugen aus verschiedenen Gründen eine andere Sprache. Insbesondere besuchen, neben lokalen Kund:innen, auch internationale Tourist:innen den Markt. Sie tendieren dazu, Englisch als Kommunikationsmedium zu nutzen. In der Tat stehen Deutsch und Englisch als Verkehrssprachen des Marktes in einem dichotomen Verhältnis zueinander. Die Verkäufer:innen und lokalen Besucher:innen des Marktes benutzen überwiegend das Deutsche zur Kommunikation, außer es gibt eine gemeinsame Heritage-Sprache wie Türkisch oder Arabisch, dann werden diese überwiegend benutzt. Die Benutzung der letztgenannten Sprachen erfolgt oft in Form eines monolingualen Gespräches. Falls sie doch im Wechsel mit dem Deutschen gesprochen werden, dann womöglich mit einer Interpretierbarkeit als ,mixed code as default", insbesondere wenn sowohl Verkäufer:innen als auch Kund:innen derselben bilingualen Sprecher:innengruppe angehören.

Im Gegensatz dazu verwenden Verkäufer:innen das Englische oft im Wechsel mit dem Deutschen. Diese CS-Konstellation kann aber nicht als „,mixed code as default“ interpretiert werden, da die Verkäufer:innen keine Zugehörigkeit zu einer deutsch-englischen bilingualen Sprecher:innengruppe aufweisen. Vielmehr hängt der Wechsel zwischen diesen beiden Sprachen mit den Englischkenntnissen der Verkäufer:innen zusammen, die nach ihren eigenen Angaben auf einem niedrigen Niveau sind (siehe (7) in Kapitel 3). Zwangsläufig zeigt sich die kommunikative und dem Kommerz dienende Motivation der Wahl des Englischen in Form von CS, so wie auch die Wahl von Sprachen, die mit der angenommenen Zugehörigkeit der Kund:innen assoziiert werden.

15 Teaser und teasen als ein dazugehöriges Verb sind Begriffe aus der Werbesprache. Duden Online führt folgende Bedeutungserklärung auf: „Kurzes Werbeelement, das die Neugier des Kunden wecken soll (z. B. ein kleiner Film, Musik- oder Textausschnitt)“ (Duden Online, s. v. Teaser). Für „teasen“ wird „locken“ als ein Synonym angegeben (ibd., s. v. teasen). 
Da das Englische zur Kommunikation mit internationalen Tourist:innen aus verschiedenen Ländern verwendet wird, also auch aus Ländern wo Englisch nicht die Hauptverkehrssprache ist, führen wir Beispiele mit dem Englischen lediglich zur Verdeutlichung des kommunikativen Faktors an. (27) zeigt einen Ausschnitt eines Verkaufsgespräches mit italienischsprachigen Kund:innen. Die Funktion des Deutschen als Verkehrssprache fällt mit der Aussage „nits verstehen“ (27:04) von K_KA_03 aus, da sie damit signalisiert, dass ihre Deutschkenntnisse eingeschränkt sind. Die Verkäuferin switcht unmittelbar ins deutsch-englische CS, damit sie von den Kund:innen verstanden werden kann. Im weiteren Verlauf der Interaktion, den wir hier der Länge wegen nicht angeführt haben, benutzt sie auch italienische Ausdrücke, um auf die Zugehörigkeit der Kund:innen $\mathrm{zu}$ referieren. (28) zeigt einen Ausschnitt eines Verkaufsgespräches mit spanischsprachigen Kund:innen. Auch hier erfolgt neben dem Englischen ein Switch ins Spanische mit ,patata patata“ (28:04), womit der Verkäufer auf die Zugehörigkeit der Kund:innen referiert.

(27) VG [deu] [eng]

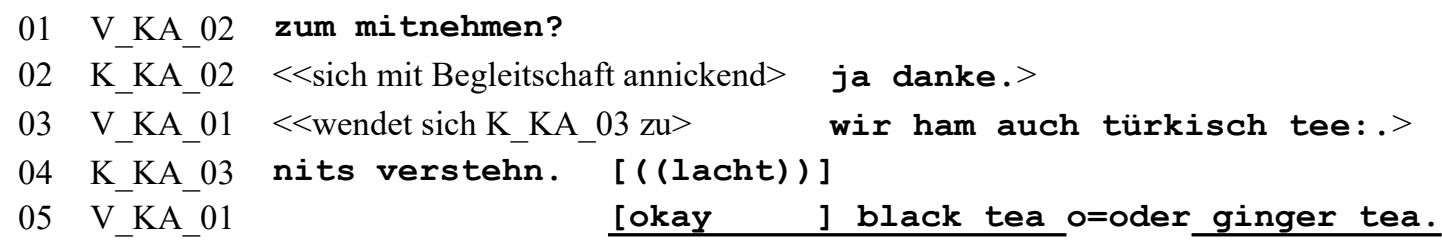

(28) VG [deu] [eng] [spa]

01 V_BR_01 HALlo.

02 K_BR_05 hello (.) eh: what are the ingredients of THIS?

03 V_BR_01 das ist eh: (-) sh=spina=cheese (.) cheese (.) eh:

04

patata=patata und $\mathrm{hm}$ ok. ( (...))

Kartoffel Kartoffel

Durch diese Sonderstellung, die das Englische einnimmt, ist es nicht einfach zu erschließen, ob die Wahl des Englischen mit der angenommen sozialen Zugehörigkeit der Kund:innen in Verbindung steht. Es gibt durchaus Beispiele, wo die Interaktion nur mit CS zwischen Deutsch und Englisch abläuft, aber das allein ist kein hinreichender Indikator für eine solche Interpretation. Nur bei Fällen, in denen es sicher ist, dass die Kund:innen eine Zugehörigkeit haben, die mit der englischen Sprache assoziiert ist, wäre diese Interpretation sinnvoll. Beispielsweise bei Tourist:innen aus England. Der kommunikative Faktor würde bei CS in solchen Verkaufsgesprächen mit dem Faktor der Identitätskonstruktion interagieren, um eine kommerzielle Motivation zu konstituieren.

\section{Fazit: CS an der Schnittstelle von Kommerz, Kommunikation und Identität}

Anhand verschiedener theoretischer Ansätze zu Identitätskonstruktion, Face-Theorie, Spracheinstellungen, Sprachkommodifizierung, CS-Forschung und mit Ansätzen der interaktionalen Linguistik wurde gezeigt, dass einigen CS-Phänomenen auf dem Markt eine kommerzielle Motivation zugeschrieben werden kann. Kommerzialität referiert hier auf das Ziel eines Verkaufsakts, also auf die grundlegende Intention der Verkäufer:innen dem Geschäftsverkehr/Kommerz nachzugehen. Durch Beispiele aus den Marktdaten wurde gezeigt, dass das kommerzielle CS durch ein komplexes Geflecht von drei Faktoren, die ineinander übergehen, interagieren oder sich gegenseitig bedingen bzw. ausschließen, näher bestimmt 
werden kann. Für die einzelnen Verkaufsinteraktionen treten einige dieser Faktoren teilweise mehr in den Vordergrund als andere, die vielleicht gar keine Rolle spielen. Abbildung 2 illustriert dieses Geflecht in Form eines Venn-Diagramms.

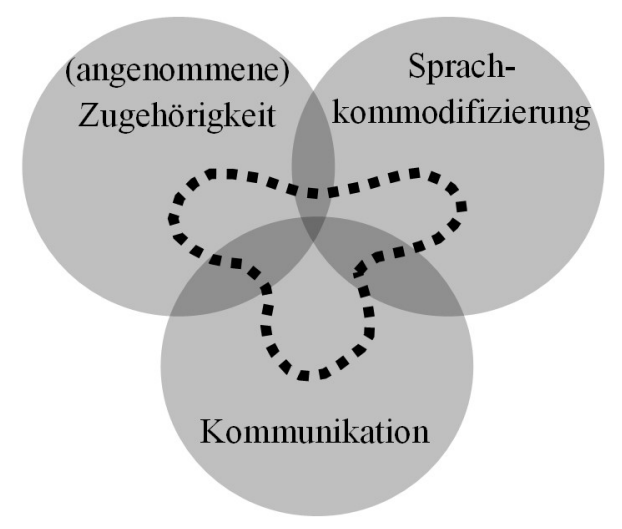

\section{Abbildung 2: Geflecht zusammenhängender Faktoren beim kommerziellen CS}

Die gestrichelten Linien verdeutlichen, dass der Bewegungsraum des kommerziellen CS dynamisch ist und vom Faktor der angenommenen Zugehörigkeit, der Sprachkommodifizierung und des kommunikativen Zwecks bedingt werden kann:

- (angenommene) Zugehörigkeit: Aus verschiedenen semiotischen Ressourcen, worunter sowohl optische als auch sprachliche Merkmale fallen, konstruieren Verkäufer:innen eine Identität der Kund:innen. Bei dieser konstruierten Identität handelt es sich um eine angenommene soziale Zugehörigkeit, die mit einer Sprache oder im weitesten Sinne einer Varietät assoziiert wird. Verkäufer:innen versuchen in den Interaktionen in dieser Varietät zu kommunizieren, was sich auf der strukturellen Oberfläche in Form von CS gestalten kann. Kund:innen fühlen sich damit besonders angesprochen oder wertgeschätzt, da dadurch zwei Fellowship-Faces bestärkt werden. Zum einen das FellowshipFace, ein Teil des Marktes zu sein und als Kund:in behandelt zu werden und zum anderen das Fellowship-Face als Sprecher:in einer Sprache.

- Sprachkommodifizierung: Einzelnen Sprachen wie Spanisch, Englisch, Französisch und Deutsch oder der Mehrsprachigkeit an sich wird von Verkäufer:innen ein kommerzieller Mehrwert zugesprochen, der auf Spracheinstellungen beruht. Dadurch werden diese Sprachen oder die Mehrsprachigkeit instrumentalisiert und wirken sich positiv auf das Geschäft aus.

- Kommunikation: Manchmal zielt der Switch in eine Sprache weder auf die soziale Zugehörigkeit der Kund:innen ab, noch auf eine Sprachkommodifizierung. CS kann essentiell für die Kommunikation sein, wenn das Gegenüber die Verkäufer:innen nur auf diese Weise versteht. Beispielsweise zeigt sich die Sprachwahl der Verkäufer:innen in Form von CS zwischen Deutsch und Englisch, wenn sie mit internationalen Tourist:innen kommunizieren.

In den Beispielen (10) bis (16) tritt die angenommene Zugehörigkeit als wichtigster Faktor in den Vordergrund. Für (13) wurde gezeigt, dass ein Verkäufer auch mit einer Sprache, die mit seiner eigenen Zugehörigkeit assoziiert wird, Nähe erzeugen kann. In den Beispielen (17) und (18) zum Marktschreien bewegt sich das CS zwischen der angenommenen Zugehörigkeit und der Sprachkommodifizierung und kann dahingehend interpretiert werden, dass beide Faktoren 
gleichzeitig auf verschiedene Kund:innen zutreffen. Für das Englische in (3), (27) und (28) tritt der kommunikative Faktor stärker in den Vordergrund als für die anderen Beispiele.

Das kommerzielle CS ist nur ein Teil der Vielfalt an CS, das auf dem Markt anzutreffen ist. Vor allem kann CS in Privatgesprächen zwischen Verkäufer:innen, oder genauer gesagt in Gesprächen, wo niemand die Rolle K annimmt, prinzipiell nicht mit Kommerzialität attribuiert werden, so wie in (29), (31) und (32) illustriert. Aber auch bei Verkaufsgesprächen mit Interaktant:innen, die derselben bilingualen Sprecher:innengruppe angehören, ist es schwer, das CS mit Kommerzialität zu erklären (30). Denn in solchen Sprecher:innengruppen hat sich so etwas wie das in den Präliminarien (Kapitel 2) beschriebene „mixed code as default“ oder „Diskursmodus“ (Poplack 2015; Lim/Ansaldo 2016; Pfaff 1979; Blommaert 2010; aber auch Translanguaging-Theorien gehen verstärkt darauf ein) entwickelt, wo Switches sehr oft durch thematische Zusammenhänge motiviert sind oder eine diskursive Motivation schwer oder nicht zielführend scheint.

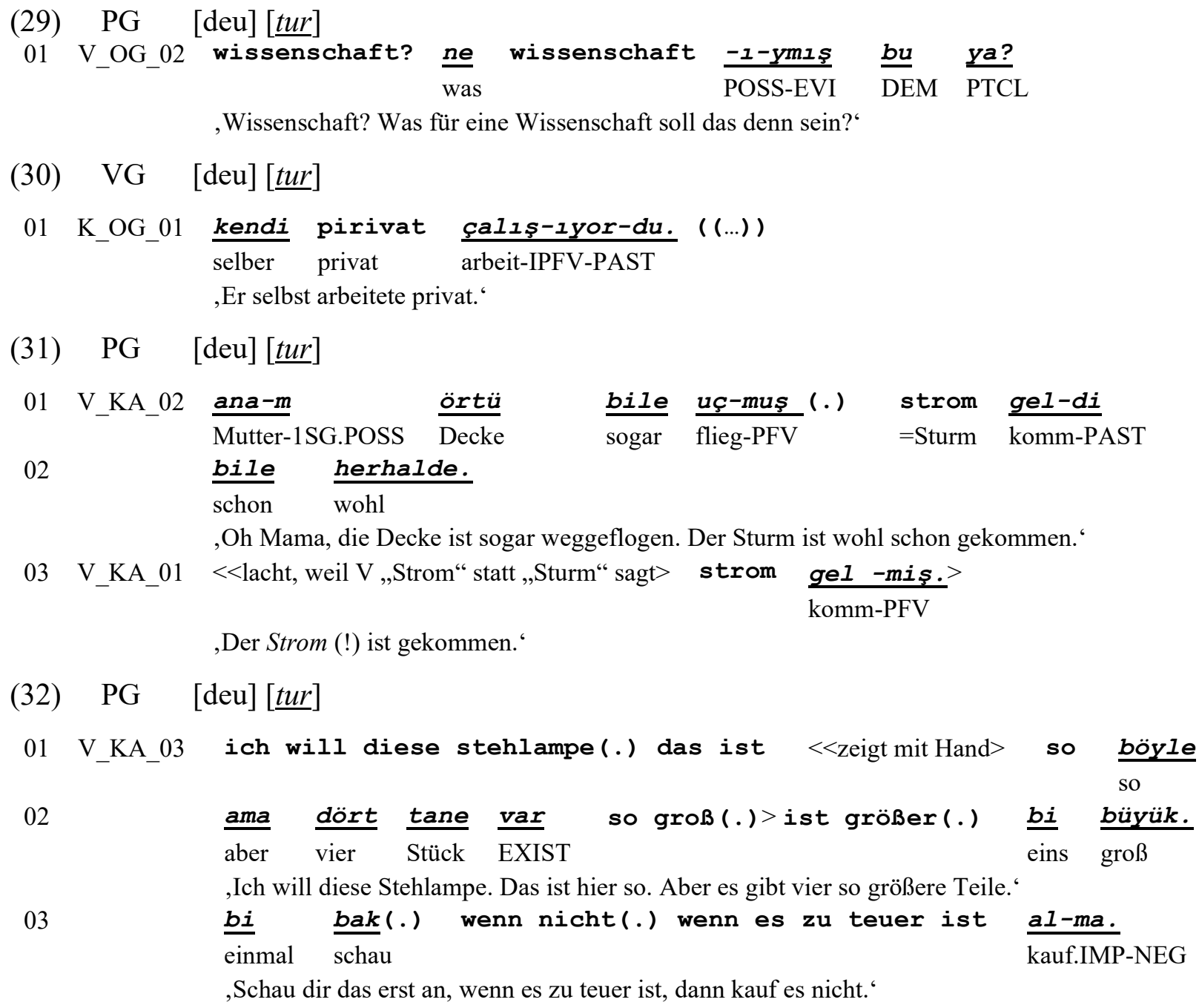

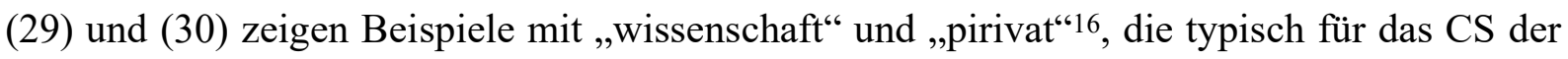
türkisch-deutschsprachigen Sprecher:innengruppe in Deutschland sind. Das sind Begriffe aus

\footnotetext{
16 Pirivat ist eine an die türkische Phonotaktik angeglichene Form von privat. Die Angleichung an die Phonotaktik einer bestimmten Sprache kann, je nach theoretischem Standpunkt, neben CS auch als Entlehnung bewertet werden.
} 
der Bildungs- und Arbeitswelt, wo Deutsch stärker gebraucht wird als andere Sprachen, weshalb es nicht verwunderlich ist, dass gerade Lexeme aus dem Bereich stärker im CS bilingualer Sprecher:innen vorkommen. In (31) stammt der Begriff „,strom“ nicht unbedingt aus einer Domäne, in der Deutsch stärker vertreten ist. Solche Switches können sich kontextbedingt entwickeln, wenn beispielsweise eine Referenz zum selben deutschen Begriff besteht. Es geht um einen spezifizierten Sturm, bile (türk. ,schon') zeigt, dass dieser, wahrscheinlich durch deutschsprachige Medien, angekündigt war. ${ }^{17}$ In (32) sehen wir eine erweiterte Form dieses „mixed code as default", wo der thematische Zusammenhang einzelner Lexeme sich für die Motivation des CS nicht als zielführend zeigt. Die Sprecherin switcht auf inter- und intraphrasaler Ebene immer wieder zwischen den beiden Sprachen und bildet komplexere Strukturen wie Bedingungssätze zweisprachig. Dies könnte in Relation zu Theorien von zusammenwirkenden Grammatiken betrachtet werden (cf. Torres Cacoullos/Travis 2015; Höder 2014, 2018). In der bisherigen CS-Forschung werden Phänomene dieser Art, die typisch für bilinguale Sprecher:innengruppen sind, eingehend beschrieben und analysiert (Beispiele gab es in Fußnote 2).

Gemäß dem „,metrolingualism“-Ansatz (Otsuji/Pennycook 2010; Pennycook/Otsuji 2015), der einen urbanen Raum als Quelle des sprachlichen Inputs definiert, gibt der Markt selbst den sprachlichen Input, was zu einer „,mehrstufigen Mehrsprachigkeit“ führt, in der verschiedene sprachliche Ressourcen mobilisiert werden. Aus einer solchen Perspektive heraus könnte man die Definition des „,mixed code as default“ von der Sprache einer bilingualen Sprecher:innengruppe auf diese „mehrstufige Mehrsprachigkeit“ ausdehnen, jedoch mit einem großen Unterschied: Das dynamische Wesen des Marktes mit immer wechselnden Besucher:innen mit verschiedenen sprachlichen Hintergründen führt eher zu einem instabileren sprachlichen Konstrukt. Wiese (2020) vergleicht die Kommunikation in einem solchen Setting mit dem, was Velupillai (2015) in einer kontaktlinguistischen Arbeit als „Jargon“ definiert. Sie analysiert die linguistische Situation unter anderem auch auf dem Maybachufer-Markt aus dieser Perspektive und beschreibt Prinzipien, die die Mechanismen eines Marktjargons festlegen:

From a contact-linguistic point of view, such multilingual practices could be seen as something
like the "jargons" described in creole studies; cf. Vellupilai (2015: 534), "individual ad hoc solu-
tions in individual contact situations leading to a highly variable and unstable contact language".
If taken literally, there is no such thing as a jargon, then, since these solutions will differ from
speaker to speaker and emerge differently in each communicative situation. However, as we will
see below for the example of two urban markets in Berlin, one can often find some recurring
patterns in these settings, and perhaps the best way to think of an urban market's jargon is as an
integrative linguistic practice characterised by (a) access to a spatially determined, but principally
open range of linguistic resources and (b) a liberal use of individual ad hoc solutions, but at the
same time (c) guided by local customs of language choice and language dominance and (d) cen-
tering around a shared core of recurring patterns.

(Wiese 2020: 268)

Klare Grenzen eines Marktjargons zu ziehen ist also nicht wirklich möglich, da es diese so nicht gibt. Besonders der Punkt (d) gibt aber einen Anhaltspunkt für eine differenziertere Herangehensweise auch im Zusammenhang mit der Bestimmung von kommerziellem CS. Eine quantitative Analyse wurde nicht durchgeführt, aber dennoch können wir sagen, dass einige Wörter

17 V_KA_02 sagt strom statt Sturm, weil sie es vielleicht so aufgefasst hat. 
auf dem Markt frequenter auftreten. Besonders zu erwähnen sind deutsche Begriffe die in die Syntax anderer Sprachen integriert werden. Beispielsweise wird Angebot in (33) in einen türkischen oder in (34) in einen arabischen Satz eingebettet. Ähnlich verhalten sich auch bitteschön (35) und bittschön (36). Aber auch Wörter für markttypische Utensilien wie Tüte (37) oder Klassifikatoren wie Schale in (16:01) werden häufig in Phrasen anderer Sprachen eingebettet.

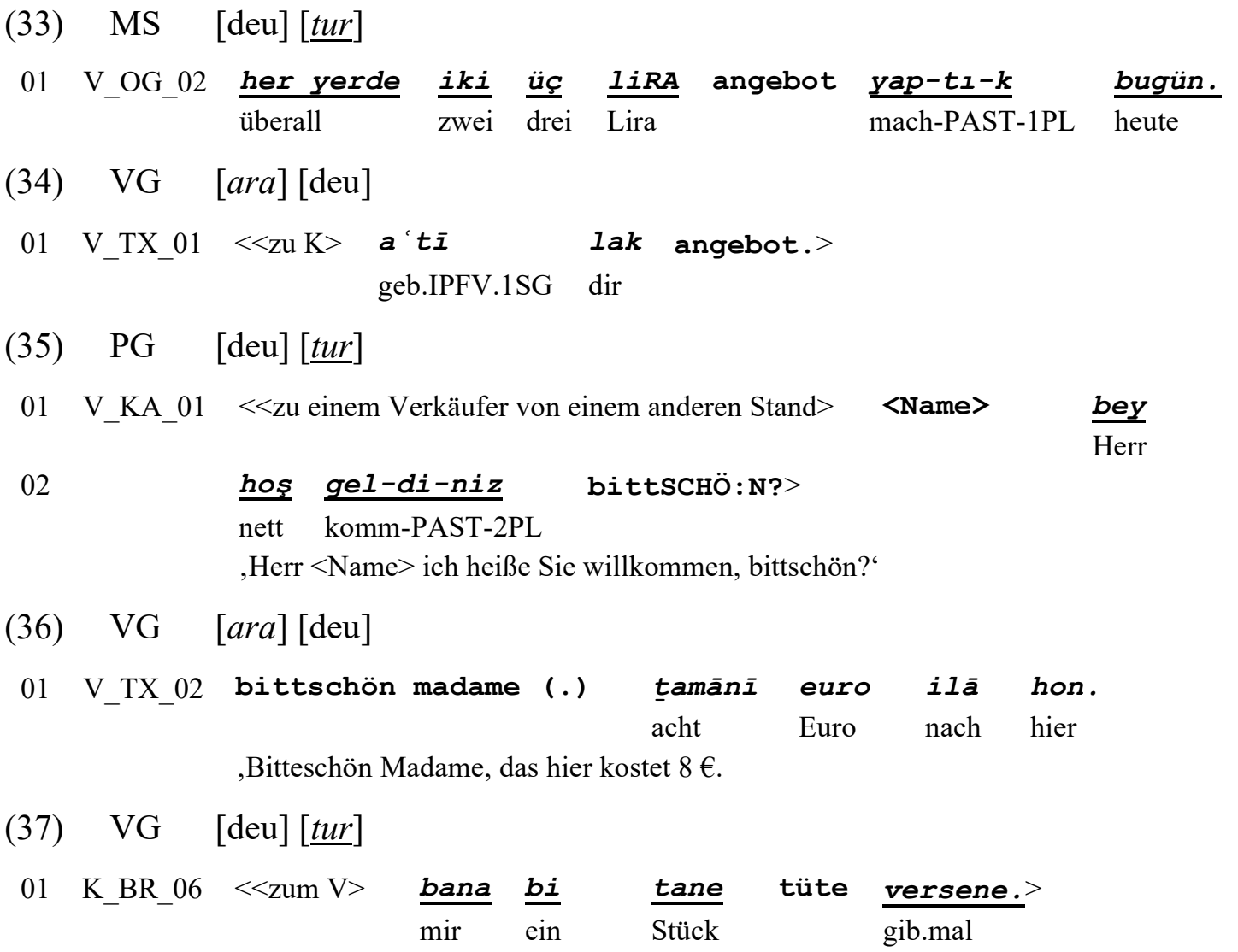

Diese Begriffe konnotieren das Marktgeschehen. Dass wir sie nicht als Beispiele kommerziellen CS nennen hat keinen motivationalen, sondern eher strukturellen Grund. Ihre hohe Frequenz, auch eingebettet in nicht-deutsche Phrasen, ermöglicht die Interpretation, dass es sich bei diesen Begriffen um mögliche Kandidaten für feste Elemente in einem Marktjargon handelt. Dieser Marktjargon ist als dynamischer Sprachgebrauch jenseits von separaten Sprachen wie Deutsch, Türkisch oder Arabisch zu verstehen. Auch Produktbezeichnungen auf dem Markt sind des Öfteren auf Deutsch. Beispielsweise sind Wörter wie Limette und Clementine nicht unüblich in großteilig türkischsprachigen Verkaufsgesprächen am Obst- und Gemüsestand oder der Schneeanzug am Textilienstand in arabischsprachigen Gesprächen.

Im Kontrast dazu stehen Anredeformen, die aus verschiedenen Sprachen stammen und in deutsche Phrasen eingebettet wurden. Sie bilden ein weiteres Feld mit Lexemen, die als Teil eines Marktjargons aufgefasst werden können, da auch sie hochfrequent auf dem Markt vorkommen. Der Unterschied ist, dass sie im Gegensatz zu den oben genannten Beispielen die Besucher:innen des Marktes persönlich ansprechen und sich auf ihre Zugehörigkeit beziehen. Deswegen sehen wir in den Beispielen (10) und (15) mit ,abla“ Anredeformen als kommerzielles CS. Die Interpretation hängt hier also von der Perspektive $a b$. 
Diese Diskussion gibt Inspirationen für weitere Forschungsfragen. Vor allem könnte eine strukturelle Analyse der Daten neue Perspektiven in die Forschung bringen, die versucht die Unterschiede zwischen CS- und Entlehnungsphänomenen zu definieren. Außerdem spielt neben der gesprochenen Sprache auch die geschriebene Sprache auf dem Markt eine wichtige Rolle. Duman (2017) und Duman und Lin (2021) zeigen eine Analyse von Schildern auf dem Maybachufer-Markt (im Vergleich zu den Schildern im Dong-Xuan-Center in Berlin-Lichtenberg) mit Werkzeugen der Linguistic-Landscape-Forschung. Die Linguistic-Landscape des Marktes weist eine Mehrsprachigkeit auf, die sich durch Übersetzungen und verschiedene Formen der Sprachmischungen zeigt. Die Mischungen zeigen sich sowohl in Form von CS in der Schriftsprache, als auch von grammatisch und graphematisch integrierten Wörtern bzw. Ausdrücken. Außerdem zeigt die Untersuchung, dass die Wahl der Sprachen sich nach den angebotenen Waren, der größeren Kund:innengruppen und den relevanten Marketingstrategien orientiert und bestimmte Sprachen zum Teil kommodifiziert werden, was aus dem Blickwinkel einer kommerziellen Motivation interessant ist.

Ein weiterer Forschungsansatz, der direkt an das Ergebnis der vorliegenden Arbeit anknüpfen würde, wäre das kommerzielle CS aus der anderen Richtung zu betrachten. In (19) haben wir gesehen, dass sich die türkischsprachigen Verkäufer:innen wertgeschätzt fühlen, wenn sie von Kund:innen auf Türkisch angesprochen werden, die diese Sprache nicht als L1 sprechen. Es wäre interessant zu verstehen, welche Prozesse sich dabei vollziehen, ob Kund:innen auch mit ähnlichen Strategien Identitäten konstruieren oder, und ganz besonders, ob sie davon einen kommerziellen Nutzen beispielsweise in Form von vergünstigten Preisen ziehen.

\section{$8 \quad$ Literaturverzeichnis}

Allen, Shanley (2009): „Typological constraints on code mixing in Inuktitut-English bilingual adults“. In: Mahieu, Marc-Antoine/Tersis, Nicole (eds.): Variations on Polysynthesis: The Eskaleut languages. Amsterdam/Philadelphia, Benjamins: 273-306.

Auer, Peter (1995): „The pragmatics of code-switching: A sequential approach“. In: Milroy, Lesley/Muysken, Pieter (eds.): One speaker two languages: Cross-disciplinary perspectives on code-switching. New York, Cambridge University Press: 115-135.

Auer, Peter (1999): „From code-switching via language mixing to fused lects: Towards a dynamic typology of bilingual speech“. International Journal of Bilingualism 3: 309-332.

Backus, Ad A. M. (1996): Two in one: Bilingual speech of Turkish immigrants in The Netherlands. Tilburg: Tilburg University Press.

Bhatia, Tej K. (1992): „Discourse functions and pragmatics of mixing: Advertising across cultures“. World Englishes 11/2-3: 195-215.

Bishop, Hywel et al. (2005): „Globalisation, advertising and language choice: shifting values for Welsh and Welshness in Y Drych, 1851-2001“. Multilingua 24/4:343-78.

Blackledge, Adrian/Creese, Angela (2010): Multilingualism: A Critical Perspective. London: Continuum.

Block, David (2018): „7. What on Earth is 'Language Commodification’?“. In: Schmenk, Barbara/ Breidbach, Stephan/Küster, Lutz (eds.): Sloganization in Language Education Discourse. Bristol, Multilingual Matters: 121-141. 
Blom, Jan-Petter/Gumperz, John Joseph (1972): „Social meaning in linguistic structure: Codeswitching in Norway“. In: Gumperz, John Joseph/Hymes, Dell (eds.): Directions in Sociolinguistics: The Ethnography of Communication. New York, Holt, Rinehart and Winston: 407-434.

Blommaert, Jan (2007): „Sociolinguistics and discourse analysis: Orders of indexicality and polycentricity“. Journal of Multicultural Discourses 2/2: 115-130.

Blommaert, Jan (2010): The Sociolinguistics of Globalization. Cambridge: Cambridge University Press.

Bourdieu, Pierre (1977): Outline of a theory of practice. Cambridge: Cambridge University Press.

Boztepe, Erman (2002): „Issues in Code-Switching: Competing Theories and Models“. Working Papers in TESOL and Applied Linguistics 3/2: 1-27.

Brown, Penelope/Levinson, Stephen C. (1987): Politeness: Some Universals in Language Usage. Cambridge: Cambridge University Press.

Bucholtz, Mary/Hall, Kira (2005): „Identity and interaction: a sociocultural linguistic approach“. Discourse Studies 7/4-5: 585-614

Bullock, Barbara E./Toribio, Almeida Jacqueline (eds.) (2009): The Cambridge Handbook of Linguistic Code-Switching. Cambridge: Cambridge University Press.

Cameron, Deborah (2012): „The commodification of language: English as a global commodity“. In: Nevalainen, Terttu/Traugott, Elizabeth Closs (eds.): The Oxford Handbook of the History of English. Oxford, Oxford University Press: 352-361.

Canagarajah, Suresh (2009): „The plurilingual tradition and the English language in South Asia“". In: Lim, Lisa/Low, Ee-Ling (eds.): Multilingual, Globalising Asia: Implications for Policy and Education, AILA Review 22: 5-22.

Canagarajah, Suresh (2013): Translingual Practice: Global Englishes and Cosmopolitan Relations. New York: Routledge.

Çetinoğlu, Özlem (2017): „A Code-Switching Corpus of Turkish-German Conversations“. Proceedings of the 11th Lunguistic Annotation Workshop. Valencia, Association for Computational Linguistics: 34-40.

Coulmas, Florian (1992): Language and economy. Oxford: Blackwell.

Creese, Angela/Blackledge, Adrian (2011): „Separate and flexible bilingualism in complementary schools: Multiple language practices in interrelationship“. Journal of Pragmatics 43: 1196-1208.

Domenici, Kathy/Littlejohn, Stephen W. (2006): Facework: Bridging theory and practice. Thousand Oaks, CA: Sage.

Duden Online: duden.de/rechtschreibung [03.05.2021].

Duman, Irem (2017): Sprachlandschaft eines urbanen Wochenmarktes: Untersuchung des Berliner Maybachufermarktes. Masterarbeit, Universität Potsdam.

Duman, Irem (2021): „Untersuchung sprachlicher Praktiken auf einem hochdiversen urbanen Wochenmarkt"“. Vortrag im 127. Forschungskolloquium Mehrsprachigkeit, Migration, Minderheiten (Soziolinguistik und Mehrsprachigkeit), 08.01.2021. Frankfurt (Oder), EuropaUniversität Viadrina.

Duman, Irem/Lin, Daiying (2021): „Linguistic Landscape im hochdiversen urbanen Raum: das linguistische Bild zweier Berliner Märkte“. In: Ziegler, Evelyn/Marten, Heiko F. (eds.): 
Linguistic Landscapes im deutschsprachigen Kontext: Forschungsperspektiven, Methoden und Anwendungsmöglichkeiten im Unterricht und Sprachmarketing. Frankfurt am Main, Lang: 165-197.

Eckert, Penelope (2005): Variation, convention, and social meaning. Paper presented at the annual meeting of the Linguistic Society of America. Oakland CA. Jan. 7, 2005. justinecassell.com/discourse09/readings/EckertLSA2005.pdf [29.11.2020].

Eckert, Penelope (2008): „Variation and the indexical field“. Journal of Sociolinguistics 12/4: 453-476.

Eckert, Penelope (2016): „Third Wave Variationism“. Oxford Handbooks Online doi: 10.1093/ oxfordhb/9780199935345.013.27.

García, Ofelia (2009a): Bilingual Education in the 21st Century: A Global Perspective. Oxford: Wiley.

García, Ofelia (2009b): „Education, multilingualism and translanguaging in the 21st century“. In: Skutnabb-Kangas, Tove/et al. (eds.): Social Justice through Multilingual Education. Bristol, Multilingual Matters: 140-158.

García, Ofelia (2013): „From diglossia to transglossia: Bilingual and multilingual classrooms in the 21st century“. In: Abello-Contesse, Christian et al. (eds.): Bilingual and Multilingual Education in the 21st Century: Building on Experience. Bristol, Multilingual Matters: 155175.

García, Ofelia (2014): „Countering the dual: Transglossia, dynamic bilingualism and translanguaging in education“. In: Rubdy, Rani/Alsagoff, Lubna (eds.): The Global-Local Interface and Hybridity: Exploring Language and Identity. Bristol, Multilingual Matters: $100-118$.

Gardner-Chloros, Penelope (1991): Language selection and switching in Strasbourg. Oxford: Oxford University Press.

Gardner-Chloros, Penelope (2009): Code-switching. New York: Cambridge University Press.

Gerritsen, Marinel et al. (2010): „English in product advertisements in non-English speaking countries of Western Europe. Product image and comprehension of the text". Journal of Global Marketing 23/4: 349-365.

Giles, Howard (ed.) (1978): Language, Ethnicity and Intergroup Relations. London: Academic Press.

Goffmann, Erving (1955): „On face-work: an analysis of ritual elements in social interaction“. Psychiatry. Journal for the Study of Interpersonal Processes 18: 213-231.

Goffmann, Erving (1967): Interaction ritual: Essays in face-to-face behavior. Chicago, IL: Aldine Publishing Company.

Gumperz, John J. (1982): Discourse Strategies. Cambridge: Cambridge University Press.

Gumperz, John J. (1996): „Introduction to Part IV2“. In: Gumperz, John J./Levinson, Stephen (eds.): Rethinking Linguistic Relativity. Cambridge, Cambridge University Press: 359-373.

Heller, Monica (2010): „The Commodification of Language“. Annual Review of Anthropology 39: $101-114$.

Heller, Monica/Duchêne, Alexandre (2016): „Treating language as an economic resource: Discourse, data and debate“. In: Coupland, Nikolas (ed.): Sociolinguistics - Theoretical Debates. Cambridge, Cambridge University Press: 139-156. 
Höder, Steffen (2014): „Constructing diasystems: Grammatical organisation in bilingual groups“'. In: Åfarli, Tor A./Mæhlum, Brit (eds.): The Sociolinguistics of Grammar. Amsterdam/Philadelphia, Benjamins: 137-152.

Höder, Steffen (2018): „Grammar is community-specific: Background and basic concepts of Diasystematic Construction Grammar". In: Boas, Hans C./ Höder, Steffen (eds.): Constructions in Contact. Constructional Perspectives on Contact Phenomena in Germanic Languages. Amsterdam/Philadelphia, Benjamins: 37-70.

Imo, Wolfgang/Lanwer, Jens Philipp (2019): Interaktionale Linguistik: Eine Einführung. Berlin: Metzler.

Holmes, Janet (2013): An Introduction to Sociolinguistics. 4. Auflage. London: Routledge.

Johnstone, Barbara/Kiesling, Scott (2008): „Indexicality and experience: Exploring the meanings of /aw/-monophthongization in Pittsburgh“. Journal of Sociolinguistics 12/1, 5-33.

Jørgensen, J. Normann (2008a): Languaging: Nine Years of Poly-lingual Development of Young Turkish-Danish Grade School Students. Kopenhagen: University of Copenhagen.

Jørgensen, J. Normann (2008b): „Polylingal languaging around and among children and adolescents“. International Journal of Multilingualism 5/3: 161-176.

Kachru, Braj (1978): „Code-mixing as a communicative strategy in India“. In: Alatis, James (ed.): Georgetown University Round Table on Languages and Linguistics. Washington DC, Georgetown University Press: 107-124.

Kachru, Braj (1983): „On mixing“. In: Kachru, Braj (ed.): The Indianization of English: The English language in India. New Delhi, Oxford University Press: 193-207.

Kallmeyer, Werner/Keim, Inken (2003): „Linguistic variation and the construction of social identity in a German-Turkish setting. A case study of an immigrant youth group in Mannheim, Germany“. In: Androutsopoulos, Jannis K. (ed.): Discourse Constructions of Youth Identities. Philadelphia, Benjamins: 29-43.

Kelly-Holmes, Helen (2000): „Bier, parfum, kaas: language fetish in European advertising“. European Journal of Cultural Studies 3/1:67-82.

Kelly-Holmes, Helen (2005): Advertising as Multilingual Communication. London: Palgrave Macmillan.

Labov, William (1963): „The social motivation of a sound change“. Word 19/3: 273-309.

Li, Wei (2011): „Moment Analysis and translanguaging space. Discursive construction of identities by multilingual Chinese youth in Britain“. Journal of Pragmatics 43: 1222-1235.

Li, Wei (2017): „Translanguaging as a Practical Theory of Language“. Applied Linguistics 39/1: 9-30.

Lim, Lisa/Ansaldo, Umberto (2016): Languages in Contact. Cambridge: Cambridge University Press.

Lim, Tae-Seop/Bowers, John Waite (1991): „Facework solidarity, approbation, and tact“. $\mathrm{Hu}$ man Communication Research 17, 415-450.

MacSwan, Jeff (2017): „A Multilingual Perspective on Translanguaging“. American Educational Research Journal 54/1: 167-201.

Makoni, Sinfree/Pennycook, Alastair (2012): „Disinventing multilingualism: From monological multilingualism to multilingual francas“. In: Martin-Jones, Marilyn/Blackledge, Adrian/ Creese, Angela (eds.): The Routledge Handbook of Multilingualism. New York, Routledge: 439-453. 
Marx, Karl (1993[1858]): Grundrisse. Harmondsworth: Penguin Classics.

Meeuwis, Michael/Blommaert, Jan (1998): „A monolectal view of coe-switching: Layered code-switching among Zairians in Belgium“. In: Auer, Peter (ed.): Code-Switching in Conversation: Language, Interaction and Identity. London, Routledge: 76-99.

Milroy, Lesley/Muysken, Pieter (eds.) (1995): One Speaker, Two Languages: Cross-Disciplinary Perspectives on Code-Switching. Cambridge: Cambridge University Press.

Møller, Janus Spindler (2008): „Polylingual performance among Turkish-Danes in late-modern Copenhagen“. International Journal of Multilingualism 5: 217-236.

Muysken, Pieter (2011): „Code-switching“. In: Mesthrie, Rajend (ed.): The Cambridge Handbook of Sociolinguistics. Cambridge, Cambridge University Press: 301-314.

Myers-Scotton, Carol (1993): Social Motivations for Codeswitching: Evidence from Africa. Oxford: Clarendon Press.

Myers-Scotton, Carol (2006): Multiple Voices: An Introduction to Bilingualism. Oxford: Blackwell.

O'Keefe, Barbara/Stephard, Gregory J. (1987): „The pursuit of multiple objectives in face-toface persuasive interaction. Effects of construct of construct differentiation on message organization“. Communication Monographs 54: 396-419.

Otsuji, Emi/Pennycook, Alastair (2010): „Metrolingualism: Fixity, fluidity and language in flux“. International Journal of Multilingualism 7: 240-254.

Otsuji, Emi/Pennycook, Alastair (2014): „Unremarkable hybridities and metrolingual practices“. In: Rubdy, Rani/Alsagoff, Lubna (eds.): The Global-Local Interface and Hybridity: Exploring Language and Identity. Bristol, Multilingual Matters: 83-99.

Özdil, Erkan (2010): Codeswitching im zweisprachigen Handeln: Sprachpsychologische Aspekte verbalen Planens in türkisch-deutscher Kommunikation. Münster: Waxmann.

Patrick, Peter L. (2004): „The Speech Community“. In: Chambers, Jack K./Trudgill, Peter/Natalie, Schilling-Estes (eds.): The handbook of language variation and change. Oxford, Blackwell: 573-597.

Pennycook, Alastair/Otsuji, Emi (2014): „Market lingos and metrolingua francas“. International Multilingual Research Journal 8/4: 255-270.

Pennycook, Alastair/Otsuji, Emi (2015): Metrolingualism: Language in the City. London: Routledge.

Pennycook, Alastair (2016): „Mobile times, mobile terms: the trans-super-poly-metro movement". In: Coupland, Nikolas (ed.): Sociolinguistics - Theoretical Debates. Cambridge, Cambridge University Press: 201-216.

Peterson, John (2016): „Multilingualism, Multilectalism and Register Variation in Linguistic Theory - Extending the Diasystematic Approach“ In: Fleischhauer, Jens/Latrouite, Anja/Osswald, Rainer (eds.): Explorations of the Syntax-Semantics Interface. Düsseldorf, Düsseldorf University Press: 109-148.

Pfaff, Carol W. (1979): „Constraints on Language Mixing: Intrasentential Code-Switching and Borrowing in Spanish/English“. Language 55/2: 291-318.

Pfaff-Czarnecka, Joanna (2011): „From 'identity' to 'belonging' in social research: plurality, social boundaries, and the politics of the self". Working Papers in Development Sociology and Social Anthropology 368. Universität Bielefeld. 
Poplack, Shana (1980): „Sometimes I'll start a sentence in Spanish y termino en español: toward a typology of code-switching“. Linguistics 18/7-8: 581-618.

Poplack, Shana (2015): „Code Switching: Linguistic“. In: Wright, James D. (ed.): International Encyclopedia of the Social \& Behavioral Sciences. 2. Auflage. Volume 3. Elsevier: 918 925.

Puntoni, Stefano et al. (2008): „Bilingualism and the emotional intensity of advertising language“. The Journal of Consumer Research 35/6: 1012-1025.

Rampton, Ben (2009): „Speech Community and Beyond“. In: Coupland, Nikolas/Jaworski, Adam (eds.): The New Sociolinguistics Reader. Macmillan, Red Globe Press: 694-713.

Rampton, Ben (2017): Crossing. Language and Ethnicity among Adolescents. Milton: Routledge.

Redmond, Mark V. (2015): „Face and Politeness Theories“. English Technical Reports and White Papers 2. lib.dr.iastate.edu/engl_reports/2 [15.6.2021].

Selting, Margret et al. (2009): „Gesprächsanalytisches Transkriptionssystem 2 (GAT 2)“. Gesprächsforschung - Online-Zeitschrift zur verbalen Interaktion 10: 353-402.

Singh, Rajendra (1985): „Grammatical constraints on code-switching: Evidence from HindiEnglish“. Canadian Journal of Linguistics 30: 33-45.

Sridhar, Shikaripur N./Sridhar, Kamal (1980): „The syntax and psycholinguistics of bilingual code-mixing“. Canadian Journal of Psychology 34: 407-416.

Steinbach, Horst-Ralf (1984): Englisches im deutschen Werbefernsehen: interlinguale Interferenzen in einer werbesprachlichen Textsorte. Paderborn: Schöningh.

Sultana, Shaila et al. (2015): „Transglossic language practices of young adults in Bangladesh and Mongolia“. International Journal of Multilingualism 12/1: 93-108.

Torres Cacoullos, Rena/Travis, Catherine E. (2015): „Gauging convergence on the ground: codeswitching in the community“. In: Travis, Catherine E./Torres Cacoullos, Rena (eds.): International Journal of Bilingualism, special issue: 365-386.

Tracy, Karen (1990): „,The many faces of facework“. In: Giles, H./Robinson, W.P (eds.): Handbook of Language and Social Psychology. West Sussex, Wiley \& Sons: 209-226.

Trudgill, Peter (1972): „Sex, covert prestige and linguistic change in the urban British English of Norwich". Language in Society 1/2: 179-195.

Velupillai, Viveka (2015): Pidgins, Creoles and Mixed Languages. Amsterdam/Philadelphia: Benjamins.

Wiese, Heike (2020): „Contact in the City“. In: Hickey, Raymond (ed.): The Handbook of Language Contact. 2. Auflage. Malden, MA, Wiley-Blackwell: 261-279.

Winford, Donald (2003): An Introduction to Contact Linguistics. Malden, MA: Wiley-Blackwell. 